\title{
Permutation complexes for profinite groups
}

Peter Symonds

\begin{abstract}
An important tool in the analysis of discrete groups of finite virtual cohomological dimension is the existence of a finite dimensional contractible CW-complex on which the group acts with finite stabilizers. We develop a purely algebraic analogue for profinite groups.

This enables us to reveal the connection between finiteness conditions on the cohomology of the group and those on the normalizers of the finite $p$-subgroups.
\end{abstract}

Mathematics Subject Classification (2000). 20J06; 20E18, $20 \mathrm{C} 07$.

Keywords. Profinite group, cohomology, permutation module.

\section{Introduction}

The main aim of this paper is to develop the cohomology theory of profinite groups along the lines of that done for discrete groups, as in the book of Brown [6] for example. Some of this was addressed by the author and Weigel in [33], but here we are particularly interested in constructing an algebraic analogue of a finite dimensional contractible space on which the group acts with finite stabilizers and in developing its consequences. The torsion free case was considered by Serre [26].

We start with a review of the basic module theory behind the cohomology of profinite groups. This originated in the work of Brumer [7], and is well explained in the books of Ribes and Zalesskii [22] and of Wilson [35], but we collect together the main properties that we will use and describe them from our point of view. This part might be of independent interest. We devote relatively little space to basic homological algebra, since this is a routine adaptation of the methods used in the discrete case once the module theory is understood.

We pay particular attention to permutation modules and the Brauer quotient, adapting some results of Swan on the cohomology of fixed point sets and work of Bouc on complexes of these modules. This enables us to formulate precisely the complexes we want and their properties. We then define the Tate-Farrell cohomology for a profinite group of finite virtual cohomological dimension and use the complexes to develop its properties. 
There are applications to questions about the finiteness of the number of conjugacy classes of finite subgroups and to the local cohomology of the cohomology of the group.

We wish to thank the referee for his patience and advice.

\section{Background}

2.1. Ambient ring. Let $R$ be a commutative local ring with maximal ideal $\mathfrak{m}$ and finite residue class field $k=R / \mathfrak{m}$ of characteristic $p$. We assume that $\operatorname{dim}_{k} \mathfrak{m} / \mathfrak{m}^{2}<$ $\infty$ and that $R$ is complete, i.e. $R \cong \lim R / \mathfrak{m}^{r}$. We give the quotients $R / \mathfrak{m}^{r}$ the discrete topology and then let $R$ have the inverse limit topology.

The obvious example is when $R$ is the $p$-adic integers, $\hat{\mathbb{Z}}_{p}$, but we also allow such things as $R=k$ or power series rings $k\left[\left[x_{1}, \ldots, x_{n}\right]\right]$.

2.2. Modules. We consider principally three categories of $R$-modules: the finite modules, $\mathcal{F}_{R}$; the pseudocompact modules, $\mathcal{C}_{R}$, which are inverse limits of modules in $\mathcal{F}_{R}$; and the discrete torsion modules, $\mathscr{D}_{R}$, which are direct limits of modules in $\mathcal{F}_{R}$. Both are given their limit topologies: (the objects of $C_{R}$ are profinite, hence compact and the objects of $\mathscr{D}_{R}$ are discrete). All morphisms are supposed to be $R$-linear and continuous and the set of all morphisms is denoted by $\operatorname{Hom}_{R}$. Both $\mathcal{C}_{R}$ and $\mathscr{D}_{R}$ are abelian categories in which the notions of kernel and cokernel are compatible with the underlying abelian group structure, and $\mathcal{C}_{R}$ has exact inverse limits and $\mathscr{D}_{R}$ has exact direct limits. (All limits will be over directed systems, although we do not assume that all the maps are epimorphisms or monomorphisms.) We will refer to modules in $\mathcal{C}_{R}$ and $\mathscr{D}_{R}$ as compact and discrete respectively.

It is possible to allow $k$ to be infinite if we are prepared to deal with pseudocompact modules as in [7].

Let $T^{\prime}$ be the injective hull of $k$ as an (abstract) $R$-module and let $T$ be its torsion submodule, i.e. the maximal submodule in $\mathscr{D}_{R}$. Equivalently $T=\operatorname{Hom}(R, \mathbb{Q} / \mathbb{Z})$. For example, if $R=k$ then $T=k$, if $R=\hat{\mathbb{Z}}_{p}$ then $T=\mathbb{Z} / p^{\infty}=p$-torsion in $\mathbb{Q} / \mathbb{Z}$.

The categories $\mathcal{C}_{R}$ and $\mathscr{D}_{R}$ are dual by $\operatorname{Hom}_{R}(-, T)$; this is an instance of Pontryagin duality. We will denote $\operatorname{Hom}_{R}(A, T)$ by $A^{*}$. Thus results for one category can be translated into results for the other. ([7], 2.3, [22], [14].)

It is sometimes also convenient to consider the category $\varepsilon_{R}$ of modules which are inverse limits of modules in $\mathscr{D}_{R}$. This contains both $\mathcal{C}_{R}$ and $\mathscr{D}_{R}$ as full subcategories and, in particular, if $R=\hat{\mathbb{Z}}_{p}$ then $\mathcal{E}_{R}$ contains $\hat{\mathbb{Q}}_{p}$, since it is the inverse limit of the system $\cdots \stackrel{p}{\rightarrow} \mathbb{Z} / p^{\infty} \stackrel{p}{\rightarrow} \mathbb{Z} / p^{\infty}$.

We can, of course, consider continuous morphisms from an object of one category of $R$-modules to an object of another. If $A \in \mathcal{C}_{R}$ and $B \in \mathcal{E}_{R}$ then $\operatorname{Hom}_{R}(A, B)$ is 
naturally an object of $\mathcal{E}_{R}$. It is in $\mathscr{D}_{R}$ if also $B \in \mathscr{D}_{R}$ and in $\mathcal{C}_{R}$ if $B \in \mathcal{C}_{R}$ and $A$ is finitely (topologically) generated.

In particular, it makes sense to claim that $\operatorname{Hom}_{R}(A, B) \cong \operatorname{Hom}_{R}\left(B^{*}, A^{*}\right), \quad A \in$ $\mathcal{C}_{R}, B \in \mathscr{D}_{R}$, and this is proved by taking the limit of the finite case.

2.3. Operations. On $\mathscr{D}_{R}$ we have the usual direct sum $\oplus$. The dual of $\oplus$ on $\mathcal{C}_{R}$ is just the product $\Pi$. Of course $\Pi$ is the same as the usual $\oplus$ when we are only taking the product of a finite number of modules, and in this case we will often write $\oplus$.

For $A, A_{i}, C_{i} \in \mathcal{C}_{R}$ and $B, B_{i} \in \mathscr{D}_{R}$ we have ([22], 5.1.4):

$$
\begin{aligned}
& \operatorname{Hom}_{R}\left(\lim _{\leftarrow} A_{i}, B\right) \cong \lim _{\longrightarrow} \operatorname{Hom}_{R}\left(A_{i}, B\right), \\
& \operatorname{Hom}_{R}\left(A, \underset{\lim }{\longrightarrow} B_{i}\right) \cong \underset{\lim }{\longrightarrow} \operatorname{Hom}_{R}\left(A, B_{i}\right), \\
& \operatorname{Hom}_{R}\left(A, \lim _{\leftarrow} C_{i}\right) \cong \operatorname{lom}_{R}\left(A, C_{i}\right),
\end{aligned}
$$

and so

$$
\begin{aligned}
& \operatorname{Hom}_{R}\left(\Pi A_{i}, B\right) \cong \oplus \operatorname{Hom}_{R}\left(A_{i}, B\right), \\
& \operatorname{Hom}_{R}\left(A, \oplus B_{i}\right) \cong \oplus \operatorname{Hom}_{R}\left(A, B_{i}\right), \\
& \operatorname{Hom}_{R}\left(A, \Pi C_{i}\right) \cong \Pi \operatorname{Hom}_{R}\left(A, C_{i}\right) .
\end{aligned}
$$

On $\mathcal{F}_{R}$ we have the usual tensor product $\otimes_{R}$. A sort of tensor product $\hat{\otimes}_{R}$ can be defined on $\mathcal{C}_{R}$ as follows. If $A=\lim A_{i}$ and $B=\lim B_{j}$ with $A_{i}, B_{j} \in \mathcal{F}_{R}$ then $A \hat{\otimes}_{R} B=\lim _{\leftarrow}\left(A_{i} \otimes_{R} B_{j}\right)$. ([18], [7])

The product $\hat{\otimes}_{R}$ is symmetric, associative and commutes with inverse limits, in particular products. It is the ordinary tensor product if one of $A$ or $B$ is finitely generated.

We define another sort of product $\check{\otimes}_{R}$ on $\mathscr{D}_{R}$ as the dual of $\hat{\otimes}_{R}$, so $A \check{\otimes}_{R} B \cong$ $\left(A^{*} \hat{\otimes}_{R} B^{*}\right)^{*}$ for $A, B \in \mathscr{D}_{R}$. Equivalently, $A \check{\otimes}_{R} B \cong \operatorname{Hom}_{R}\left(A^{*}, B\right)$. This product commutes with direct limits.

This is not the usual tensor product, since $\mathbb{Z} / p^{\infty} \otimes_{\hat{\mathbb{Z}}_{p}} \mathbb{Z} / p^{\infty}=0$ but $\mathbb{Z} / p^{\infty} \check{\bigotimes}_{\hat{\mathbb{Z}}_{p}}$ $\mathbb{Z} / p^{\infty} \cong \mathbb{Z} / p^{\infty}$.

$\mathcal{C}_{R}$ satisfies a form of Nakayama's Lemma, that if $U \leq V$ is closed and $U+\mathfrak{m} V=$ $V$ then $U=V$. The dual statement also holds ([7], 1.4). Notice that $U$ does not need to be topologically finitely generated.

For any $D \in \mathscr{D}_{R}$, soc $D=\bigoplus_{I} k$ for some indexing set $I$ and hence the injective hull of $D$ is $\bigoplus_{I} T$. By duality, the projective cover of $C \in \mathcal{C}_{R}$ is the same as the projective cover of $C / \operatorname{rad} C \cong \Pi_{I} k$, which is $\Pi_{I} R$.

As a consequence, every projective in $\mathcal{C}_{R}$ is of the form $\Pi_{I} R$ and there are enough projectives, and dually for $\mathscr{D}_{R}$.

However, $\Pi_{I} \hat{\mathbb{Z}}_{p}$ is not always projective in the category of abstract $\hat{\mathbb{Z}}_{p}$-modules; see [16]. 
2.4. Modules for groups. If $G$ is a profinite group then we can consider the categories consisting of objects in one of the categories above endowed with a continuous action of $G$, and we denote these by $\mathcal{C}_{R}(G), D_{R}(G)$ and $\mathcal{E}_{R}(G)$. The morphisms will be denoted by $\operatorname{Hom}_{G}$. All the constructions and identities above are natural, hence equivariant, and so yield versions for these new categories. $\mathcal{C}_{R}(G)$ and $\mathscr{D}_{R}(G)$ are still abelian categories and Pontryagin dual, but of course we take the contragredient module to ensure that all our modules are left $G$-modules.

Every object in $\mathcal{C}_{R}(G)$ is, in fact, an inverse limit of objects in $\mathcal{F}_{R}(G)$, and similarly for $\mathcal{D}_{R}(G)$ and $\mathcal{E}_{R}(G)$ ([22], 5.3.3, [18], II 2.2.6). They both satisfy a version of the Krull-Schmidt property, that if $M \in \mathcal{C}_{R}(G)$ is a product of indecomposable modules (or $N \in \mathscr{D}_{R}(G)$ is a sum of indecomposable modules) with local endomorphism rings then this decomposition is essentially unique ([14]).

Recall that being indecomposable is equivalent to having a local endomorphism ring for finitely generated $R[F]$-modules when $F$ is a finite group. For $M \in \mathcal{C}_{R}(G)$ and $N$ an open normal subgroup of $G$ let $M_{N}$ denote the module of coinvariants, i.e. the largest continuous quotient on which $N$ acts trivially; it is a $G / N$-module.

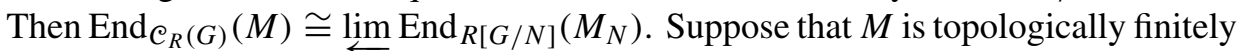
generated and, for each open normal subgroup $N<G$, there is an open normal subgroup $N^{\prime}$ such that $N^{\prime}<N$ and $M_{N^{\prime}}$ is indecomposable as a $G / N^{\prime}$-module. Then $M$ is an indecomposable module with local endomorphism ring.

Suppose that $G=\lim G / N_{i}$ for some system $N_{i}$ of open subgroups. Then we define the complete group algebra $R[[G]] \in \mathcal{C}_{R}$ by $R[[G]]=\lim R\left[G / N_{i}\right]$. An object of $\mathcal{E}_{R}$ has a continuous action of $G$ if and only if it has a continuous action of $R[[G]]$. This implies that, as a left module over itself, $R[[G]]$ is projective in $\mathcal{C}_{R}(G)$. The dual of $R[[G]]$ is $F(G)$, the functions on $G$ (see $\S 3$ for more details).

Again there is a form of Nakayama's Lemma. We let $\operatorname{rad} R[[G]]$ denote the intersection of the maximal two-sided ideals of $R[[G]]$. Then if $V \in \mathcal{C}_{R}(G)$ and $U \leq V$ is closed such that $U+(\operatorname{rad} R[[G]]) V=V$ then $U=V([7], 1.4)$.

We can define $A \hat{\otimes}_{R[[G]]} B$ for $A, B \in \mathcal{C}_{R}(G)$ either as $\lim \left(A_{i} \otimes_{R G} B_{j}\right)$, where $A=\lim A_{i}$ and $B=\lim _{\leftarrow} B_{j}$ with $A_{i}, B_{j} \in \mathscr{F}_{R}(G)$, or simply as the coinvariants of $A \overleftarrow{\otimes}_{R} B$ under the diagonal action of $G$. Similarly we can define $C \check{\otimes}^{G} D$ for $C, D \in \mathscr{D}_{R}(G)$ to be the invariants of $C \check{\otimes}_{R} D$.

Induction on $\mathcal{C}_{R}(G)$ and coinduction on $\mathscr{D}_{R}(G)$ are defined by $\operatorname{Ind}_{H}^{G} M=$ $R[[G]] \hat{\otimes}_{R[[H]]} M$ and $\operatorname{Coind}_{H}^{G} N=F(G) \check{\otimes}^{H} N$. (The latter is called induced by Serre [27].) They are exact, dual to each other, and satisfy the customary adjointness properties ([22], 6.10):

$$
\begin{aligned}
\operatorname{Hom}_{G}\left(\operatorname{Ind}_{H}^{G} M, N\right) \cong & \operatorname{Hom}_{H}\left(M, \operatorname{Res}_{H}^{G} N\right), \\
& M \in \mathcal{C}_{R}(H), N \in \mathcal{C}_{R}(G) \text { or } D_{R}(G) ;
\end{aligned}
$$




$$
\begin{aligned}
\operatorname{Hom}_{G}\left(M, \operatorname{Coind}_{H}^{G} N\right) \cong & \operatorname{Hom}_{G}\left(\operatorname{Res}_{H}^{G} M, N\right), \\
& M \in \mathcal{C}_{R}(G) \text { or } D_{R}(G), N \in D_{R}(H) .
\end{aligned}
$$

As a consequence, restriction to a subgroup preserves projectives in $\mathcal{C}_{R}(G)$ and injectives in $\mathscr{D}_{R}(G)$. Also Ind preserves projectives and Coind preserves injectives. This makes it easy to show that $\mathcal{C}_{R}(G)$ has enough projectives and $\mathscr{D}_{R}(G)$ has enough injectives (see also 2.5 below).

There are the usual formulas $\operatorname{Ind}_{H}^{G} \operatorname{Res}_{H}^{G} M \cong R[[G / H]] \hat{\otimes}_{R} M$ and its dual $\operatorname{Coind}_{H}^{G} \operatorname{Res}_{H}^{G} M \cong F(G / H) \check{\otimes}_{R} M$.

2.5. Projectives. The following are equivalent for an object $P$ of $\mathcal{C}_{R}(G)$ ([7], 2.2,

3.6):

- (i) $P$ is projective in $\mathcal{C}_{R}(G)$,

- (ii) $\operatorname{Hom}_{G}(P,-)$ is exact on $\mathscr{D}_{R}(G)$,

- (iii) $\operatorname{Hom}_{G}(P,-)$ is exact on $\mathcal{F}_{R}(G)$.

The dual formulation is also true. From the formulas in 2.3, we see that projectives are preserved under products.

Since $\mathcal{E}_{R}(G)$ is not abelian, the question of whether $\operatorname{Hom}_{G}(P,-)$ is exact is more involved, even when $P$ is projective in $\mathcal{C}_{R}(G)$. First note that, since $P$ is a summand of a module induced from the trivial subgroup, it is enough to consider the case without group action. Let $A \stackrel{i}{\rightarrow} B \stackrel{j}{\rightarrow} C$ be a sequence in $\varepsilon_{R}$ that is short exact as a sequence of abelian groups. If we want it to remain exact after applying $\operatorname{Hom}_{R}(P,-)$ then we need $i$ to induce a homeomorphism between $\mathrm{A}$ and its image under the subspace topology. This is sufficient if $P$ is finitely generated. Otherwise we also need every compact submodule of $C$ to be the image of a compact submodule in $B$, which is guaranteed under several general conditions, e.g. $A$ compact, the sequence split over $R$, or $R$ a discrete valuation ring and $B$ a finite dimensional vector space over the quotient field.

A useful result is that if $A \in \mathcal{C}_{R}(G)$ and $A \cong \lim A_{i}$ for $A_{i} \in \mathcal{C}_{R}(G)$ such that for each $A_{i}$ there is an open normal subgroup $N_{i}<\overleftarrow{G}$ which acts trivially on $A_{i}$ and such that $A_{i}$ is projective over $G / N_{i}$, then $A$ is projective. ([7], 3.3, but surjections are not necessary everywhere: compare $\mathrm{A} 3$ there with 2.3.)

Each module $D \in \mathscr{D}_{R}(G)$ has an injective hull $I_{D} \in \mathscr{D}_{R}(G)$. This can be seen as follows [8]. Let $I$ be the injective hull of $D$ as an abstract $R G$-module, and let $I_{D}$ be its smallest submodule in $\mathscr{D}_{R}(G)$, i.e. the set of elements that are $\mathfrak{m}$-torsion and such that the stabilizer in $G$ is open. It is straightforward to see that this has the correct properties. Taking injective hulls commutes with $\oplus$, by 2.3 .

Dually, every $C \in \mathcal{C}_{R}(G)$ has a projective cover $P_{C} \in \mathcal{C}_{R}(G)$.

Let $S \in \mathcal{C}_{R}(G)$ be simple and hence finite; let $P_{S}$ be its projective cover. From the definition of projective cover it follows that the non-isomorphisms in $\operatorname{End}_{\mathcal{C}_{R}(G)}\left(P_{S}\right)$ 
form an ideal, so $\operatorname{End}_{\mathcal{C}_{R}(G)}\left(P_{S}\right)$ is local. Let $N$ be a normal open subgroup of $G$ that acts trivially on $S$ and let $P_{S, N}$ be the be the module of coinvariants, as in 2.4. Then $P_{S, N}$ is projective over $R[G / N]$ and there is still a surjection $P_{S, N} \rightarrow S$. But the natural map $\operatorname{End}_{\mathfrak{C}_{R}(G)}\left(P_{S}\right) \rightarrow \operatorname{End}_{\mathcal{C}_{R}(G / N)}\left(P_{S, N}\right)$ is surjective, so $\operatorname{End}_{\mathfrak{C}_{R}(G / N)}\left(P_{S, N}\right)$ is local and $P_{S, N}$ is the projective cover of $S$ as an $R[G / N]$-module.

By a similar argument, each projective in $\mathcal{C}_{k}(G)$ is the reduction modulo $\mathfrak{m}$ of one in $\mathcal{C}_{R}(G)$.

The socle of any $D \in \mathscr{D}_{R}(G)$ is of the form $\bigoplus_{i \in I} S_{i}$, where the $S_{i}$ are simple modules. Thus $I_{D} \cong \bigoplus_{i \in I} I_{S_{i}}$. Dually, for $C \in \mathcal{C}_{R}(G)$ we have $P_{C} \cong \prod_{i \in I} P_{S_{i}}$, where $C / \operatorname{rad}(C) \cong \prod_{i \in I} S_{i}$. This expression is unique by 2.4 and every projective has this form.

2.6. Cohomology. We define $\operatorname{Ext}_{G}^{*}(A, B)$ on $\mathcal{C}_{R}(G) \times \mathscr{D}_{R}(G)$ in the usual way, using either a projective resolution of $A$ or an injective resolution of $B$. It naturally takes values in $\mathscr{D}_{R}$, is balanced and has long exact sequences in either variable. We will write $\operatorname{Ext}_{R[[G]]}$ if we need to emphasize the role of $R$. This $\operatorname{Ext}_{G}^{*}$ is continuous in the sense that

$$
\begin{aligned}
& \operatorname{Ext}_{G}^{*}\left(\lim _{\leftarrow} A_{i}, B\right) \cong \lim _{\longrightarrow} \operatorname{Ext}_{G}^{*}\left(A_{i}, B\right), \\
& \operatorname{Ext}_{G}^{*}\left(A, \mathfrak{l i m}_{\longrightarrow} B_{i}\right) \cong \underset{\lim _{\longrightarrow}}{\operatorname{Ext}_{G}^{*}\left(A, B_{i}\right)}
\end{aligned}
$$

(see [7], 3.4, [22], 6.5.3). In fact, $\operatorname{Ext}_{G}^{*}(A, B)$ can be defined on $\mathscr{D}_{R}(G) \times \mathscr{D}_{R}(G)$, by using an injective resolution of $B$, or on $\mathcal{C}_{R}(G) \times \mathcal{C}_{R}(G)$, by using a projective resolution of $A$. In these cases it just takes values in abelian groups. Slightly more generally, $\operatorname{Ext}_{G}^{*}(A, B)$ can even be defined on $\mathcal{C}_{R}(G) \times \mathcal{E}_{R}(G)$, by using a projective resolution of $A$. Again it just takes values in abelian groups, although it will take values in $\mathcal{C}_{R}$ if $A$ is of type $\mathrm{FP}_{\infty}$ in $\mathcal{C}_{R}(G)$ and $B \in \mathcal{C}_{R}(G)$. If these conditions hold then we also have by [33], 3.7.2 that

$$
\operatorname{Ext}_{G}^{*}\left(A, \underset{\leftarrow}{\lim } C_{i}\right) \cong \lim _{\longleftarrow} \operatorname{Ext}_{G}^{*}\left(A, C_{i}\right) .
$$

We obtain long exact sequences in the second variable if the conditions of 2.5 are satisfied, e.g. if $A$ is of type $\mathrm{FP}_{\infty}$ or the terms in the second variable are compact. We can define restriction maps from $\mathrm{Ext}_{G}^{*}$ to $\mathrm{Ext}_{H}^{*}$ in the usual way for any closed subgroup $H$, using the fact that projective modules remain projective after restriction of the group (2.4).

There is also a derived functor $\operatorname{Tor}_{*}^{G}$ defined on $\mathcal{C}_{R}(G) \times \mathcal{C}_{R}(G)$ and taking values in $\mathcal{C}_{R}$. By duality, $\operatorname{Tor}_{r}^{G}(A, B)^{*} \cong \operatorname{Ext}_{G}^{r}\left(A, B^{*}\right)$.

The cohomology of the group is defined to be $H^{*}(G, M)=\operatorname{Ext}_{G}^{*}(R, M)$. There are the usual bar resolutions, which can be used to show that if $M \in \mathscr{D}_{R}(G)$ then $H^{*}(G, M) \cong \lim _{\longrightarrow} H^{*}\left(G / N, M^{N}\right)$ as $N$ runs through the open normal subgroups of $G$ and the maps are inflations ([22], 6.5.6). 
Cup products can be defined in the usual way, by taking the tensor product of the projective resolutions.

The usual Eckmann-Shapiro isomorphisms hold:

$$
\operatorname{Ext}_{G}^{*}\left(\operatorname{Ind}_{H}^{G} M, N\right) \cong \operatorname{Ext}_{H}^{*}\left(M, \operatorname{Res}_{H}^{G} N\right), \quad M \in \mathcal{C}_{R}(H), N \in \mathcal{C}_{R}(G) \text { or } D_{R}(G) ;
$$

$\operatorname{Ext}_{G}^{*}\left(M, \operatorname{Coind}_{H}^{G} N\right) \cong \operatorname{Ext}_{H}^{*}\left(\operatorname{Res}_{H}^{G} M, N\right), \quad M \in \mathcal{C}_{R}(G)$ or $D_{R}(G), N \in D_{R}(H)$.

There is a Lyndon-Hochschild-Serre spectral sequence $H^{p}\left(G / H, H^{q}(H, M)\right) \Rightarrow$ $H^{p+q}(G, M)$ for any closed normal subgroup $H$ and $M \in D_{R}(G)$ ([22], 7.2.4). We can also allow $M \in \mathcal{C}_{R}(G)$ if $G$ is of type $\mathrm{FP}_{\infty}$ and $H$ is open ([33], 4.2.6).

\section{Permutation modules}

Let $X$ be a profinite space and $M$ a discrete torsion $R$-module. Define $F(X, M)$ to be the $R$-module of continuous functions $X \rightarrow M$. We write $F(X)$ for $F(X, T)$.

If $G$ is a profinite group that acts continuously on $X$ then $X$ can, in fact, be expressed as the inverse limit of finite $G$-sets ([22], 5.6.4).

If $G$ also acts continuously on $M$ then $G$ acts continuously on the discrete torsion module $F(X, M)$ by $(g f)(x)=g f\left(g^{-1} x\right), g \in G, f \in F(X, M), x \in X$.

Definition 3.1. A discrete permutation $G$-module is an $F(X)$ as above. An $R$ permutation module is a summand of a permutation module. A compact $(R)$-permutation module is the Pontryagin dual of a discrete one and we write $R[[X]]=F(X)^{*}$.

If $H \leq G$ also acts on the right of $X$ and $M$ is an $H$-module, then $F(X, M)$ is a $G \times H$-module by $((g, h) f)(x)=h f\left(g^{-1} x h\right) g \in G, h \in H, f \in F(X, M)$, $x \in X$.

If $X$ and $M$ are finite then $F(X, M) \cong F(X) \check{\otimes}_{R} M$, where $H$ has the diagonal action and $G$ acts trivially on $M$. By taking direct limits we see that in general $F(X, M) \cong F(X) \check{\otimes}_{R} M$.

If we take $X=G$ in the above, with the usual left and right actions by multiplication, then the submodule of $H$-fixed points $F(G, M)^{1 \times H}$ is isomorphic to the coinduced $G$-module $\operatorname{Coind}_{H}^{G} M$.

Note that if $H \leq G$ and $X$ is an $H$-set then $F\left(G \times_{H} X\right) \cong \operatorname{Coind}_{H}^{G} F(X)$.

We only ever consider closed subgroups of $G$, so the notation $H<G$ will imply that $H$ is a closed subgroup. We write $H<_{o} G$ to mean that $H$ is an open subgroup of $G$ (so also closed). Similarly $H \triangleleft_{o} G$ means that $H$ is open and normal in $G$. If $M$ is a $G$-module and $H<_{o} G$ then we define $\operatorname{tr}_{H}^{G}: M^{H} \rightarrow M^{G}$ by $\operatorname{tr}_{H}^{G}(m)=$ $\sum_{g \in G / H} g m, m \in M$. Dually, we let $M_{G}$ denote the module of coinvariants and define $\operatorname{cor}_{H}^{G}: M_{G} \rightarrow M_{H}$ by $\operatorname{cor}_{H}^{G}(m)=\sum_{g \in H \backslash G} g m, m \in M$. 
Definition 3.2 (Brauer Construction). If $M$ is a (topological) $G$-module and $P \leq G$, define $M^{[P]}=k \otimes\left(M^{P} / \overline{\sum_{Q \varsubsetneqq o} P} \operatorname{tr}_{Q}^{P} M^{Q}\right)$, where the summation is over all proper open subgroups of $P$. Thus $M^{[P]}$ is a module for $N_{G}(P) / P$ over $k$. Normally we will have $M \in \mathcal{C}_{R}(G)$, in which case $M^{[P]} \in \mathcal{C}_{k}\left(N_{G}(P) / P\right)$. Sometimes $M^{[P]}$ is referred to as the Brauer quotient.

Dually, for $N \in \mathscr{D}_{R}(G)$ we set $N_{[P]}=k \check{\otimes}\left(\cap_{Q ł o} P \operatorname{ker}\left(\operatorname{cor}_{Q}^{P}: M_{P} \rightarrow M_{Q}\right)\right) \in$ $\mathscr{D}_{k}\left(N_{G}(P) / P\right)$.

In all cases the definition extends to morphisms in the obvious way, yielding a functor.

Remark. (1) Our definition for $\mathcal{C}_{R}(G)$ is not quite the usual one because we reduce modulo $\mathfrak{m}$. As a consequence $M^{[1]}=k \otimes_{R} M$.

(2) The two definitions will not always agree on $\mathcal{F}_{R}(G)$. We will apply the Brauer construction only to $R$-permutation modules, where the problem does not arise, in view of 3.5 and 3.6.

Lemma 3.3. Let $G$ be a profinite group and $H$ a closed subgroup; all limits are over $G$-equivariant directed systems.

(1) $\left(\lim _{\longrightarrow} M_{i}\right)_{[H]} \cong \lim _{\longrightarrow}\left(M_{i[H]}\right)$ and $\left(\lim _{\longrightarrow} M_{i}\right)^{[H]} \cong \lim _{\longrightarrow}\left(M_{i}^{[H]}\right)$, where the $M_{i}$ are discrete.

(2) $\left(\lim _{\leftarrow} M_{i}\right)^{[H]} \cong \lim \left(M_{i}^{[H]}\right)$, where the $M_{i}$ are compact.

(3) The Brauer construction commutes with products $\Pi$ for compact modules and with direct sums $\oplus$ for discrete modules.

(4) $\left(M^{*}\right)_{[H]} \cong\left(M^{[H]}\right)^{*}$, where $M \in \mathcal{C}_{R}(G)$ and $*$ denotes the Pontryagin dual.

Proof. (1) and (2) follow easily from the definitions and (3) is a special case of them. (4) also follows from the definitions.

The importance of the Brauer construction is that it allows us to recover $F\left(X^{P}\right)$ from $F(X)$, as is well known in the finite case (see e.g. [5]).

Lemma 3.4. If $X$ is a profinite space on which $G$ acts continuously and $P \leq G$ is a pro-p subgroup then $F(X)_{[P]} \cong k \check{\otimes}_{R} F\left(X^{P}\right)$ as discrete $N_{G}(P) / P$-modules and, dually, $R[[X]]^{[P]} \cong k\left[\left[X^{P}\right]\right]$.

Proof. This is well known when $X$ is finite, in which case the action of $G$ factors through that of a finite group. Let $X=\lim _{\leftarrow} X_{i}$. Then $F\left(X^{P}\right) \cong F\left(\lim X_{i}^{P}\right) \cong$ $\stackrel{\lim }{\longrightarrow} F\left(X_{i}^{P}\right) \cong \lim _{\longrightarrow}\left(F\left(X_{i}\right)_{[P]}\right) \cong\left(\lim _{\longrightarrow} F\left(X_{i}\right)\right)_{[P]} \cong F(X)_{[P]}$, by $3.3(1)$ 
Lemma 3.5. For an R-permutation module $M$ over a pro- $p$ group $P$ and $Q \triangleleft P$ we have $\left(M^{[Q]}\right)^{[P / Q]} \cong M^{[P]} \cong\left(k \otimes_{R} M\right)^{[P]}$ if $M$ is compact and $\left(M_{[Q]}\right)_{[P / Q]} \cong$ $M_{[P]} \cong\left(k \check{\otimes}_{R} M\right)_{[P]}$ if $M$ is discrete.

Proof. This follows from 3.4.

Lemma 3.6. If $P$ is a finite $p$-group and $M \in \mathcal{D}_{k}(P)$ is a k-permutation module then there is a natural isomorphism $M^{[P]} \cong M_{[P]}$, i.e. the two sides are isomorphic as functors on the full subcategory of $k$-permutation modules.

Proof. Let us write $[m]_{P}$ to denote the class of $m \in M$ in $M_{P}$. We claim that the assignment $m \mapsto[m]_{P}$ for $m \in M^{P}$ induces a map $\phi: M^{[P]} \rightarrow M_{[P]}$.

First we need to check that $[m]_{P}$ is in the kernel of $\operatorname{cor}_{Q}^{P}$ for $m \in M^{P}$. But $\operatorname{cor}_{Q}^{P}\left([m]_{P}\right)=\left[\sum_{g \in Q \backslash P} g m\right]_{Q}=[|P / Q| m]_{Q}=0$.

Second we need to check that $\left[\operatorname{tr}_{Q}^{P} m\right]_{P}=0$ for $m \in M^{Q}$. But again the left hand side is just $|P / Q|[m]_{P}=0$.

It is easy to check that $\phi$ is an isomorphism when $M=F(P / Q, k)$, hence also when $M=F(X, k)$, where $X$ is finite. The case of general $M$ follows by taking direct limits. Finally, the naturality gives the result for $k$-permutation modules.

Lemma 3.7. If $H, K<G$ and $H$ is finite then $F(G / H)_{[K]}$ is isomorphic to some $F(X, k)$, where $X$ is a profinite $N_{G}(K)$-set with a finite number of orbits. The stabilizers are of the form $\left(N_{G}(K) \cap{ }^{g} H\right) / K$ for $g \in G$ such that $K \leq{ }^{g} H$.

Proof. Notice that $F(G / H)_{[K]} \cong k \otimes_{R} F\left((G / H)^{K}\right) \cong F\left((G / H)^{K}, k\right)$, and $(G / H)^{K}$ consists of the cosets $g H$ such that $K^{g} \leq H$. Thus the stabilizers have the form claimed.

Also $N_{G}(K) \backslash(G / H)^{K}$ is finite because it is in bijection with the conjugacy classes of subgroups of $H$ whose members are conjugate to $K$ in $G$, by the map $N_{G}(K) g H \mapsto K^{g}$.

Lemma 3.8. Let $G$ be an (abstract) group and suppose that we have subgroups $H \triangleleft G$ and $S \leq G$ such that $S \cap H=1$ and also $P \leq G / H$. Let $\S_{H}(P)$ denote the set of subgroups $Q \leq G$ such that $Q \cap H=1$ and $Q H / H=P$; $H$ acts on this by conjugation. Then we have

$$
(G / H S)^{P} \cong \amalg_{Q \in S_{H}(P) / H} N_{H}(Q) \backslash(G / S)^{Q} .
$$

Proof. Let $\rho: G \rightarrow G / H$ and $\sigma: G / S \rightarrow G / H S$ be the quotient maps. Let $Y=$ $\sigma^{-1}\left((G / H S)^{P}\right)$, so that $(G / H S)^{P} \cong H \backslash Y$.

Notice that $Y=\left\{g S \mid P \leq \rho\left({ }^{g} S\right)\right\}$. We claim that $Y=\amalg_{\delta_{H}(Q)}(G / H S){ }^{Q}$; the result follows. 
First we show that the $(G / S)^{Q}$ are disjoint for different $Q$. If $g S \in(G / S)^{Q_{1}} \cap$ $(G / S)^{G_{2}}$ then $g S \in(G / S)^{\left\langle Q_{1}, Q_{2}\right\rangle}$ and so $\left\langle Q_{1}, Q_{2}\right\rangle \leq{ }^{g} S$. Now $\left\langle Q_{1}, Q_{2}\right\rangle \cap H \leq$ ${ }^{g} S \cap H=1$, so $\rho$ restricted to $\left\langle Q_{1}, Q_{2}\right\rangle$ is injective. But $\rho\left(\left\langle Q_{1}, Q_{2}\right\rangle\right)=P$, so $Q_{1}=Q_{2}$.

Now if $g S \in(G / S)^{Q}$ then $Q \leq{ }^{g} S$, so $P \leq \rho\left({ }^{g} S\right)$ and $g S \in Y$. Conversely, if $g S \in Y$ then let $Q=\left(\rho \mid g_{S}\right)^{-1}(P)$ and we find that $g S \in(G / S)$.

Corollary 3.9. In the circumstances of the previous lemma, but with $G$ profinite, $H$ open and $P$ a p-group we have

$$
F(G / H S)_{[P]} \cong \bigoplus_{Q \in S_{H}(P) / H}\left(F(G / S)_{[Q]}\right)^{N_{H}(Q)},
$$

where the sum has only a finite number of non-zero terms.

Proof. This follows from 3.8 and 3.4. Note that, since $H$ is open in $G$, both sides are finite dimensional after applying $k \check{\otimes}$.

Lemma 3.10. If $G$ acts continuously on the profinite set $X$ and $X$ is finitely generated under this action then we can write $X \cong \bigsqcup_{i=1}^{n} G / S_{i}$ for some finite collection of closed subgroups $S_{i}$. We have $F(X) \cong \bigoplus_{i=1}^{n} F\left(G / S_{i}\right)$.

Proof. Each orbit is both open and closed.

We want to generalize the above observation.

Suppose that $G$ acts continuously on $X$ and that $* \in X$ is a fixed point. We say that $G$ acts freely on $(X, *)$ if it acts freely on $X \backslash\{*\}$.

If $Y \subseteq X$ is closed, we define $F(X, Y)$ to be the kernel of restriction $F(X) \rightarrow$ $F(Y)$, i.e, those functions that vanish on $Y$. Note that $F(X, Y) \cong F(X / Y, *)$ (where * denotes the image of $Y)$. We write $R[[X, Y]]$ for the dual of $F(X, Y)$.

Lemma 3.11. If $G$ acts freely on $(X, *)$ then $F(X, *)$ is injective as a discrete $G$ module, hence isomorphic to a summand of a sum of $F(G)$ 's. Dually, $R[[X, *]]$ is projective as a compact $G$-module and is isomorphic to a summand of a product of $R[[G]]$ 's.

Proof. (cf. [22], 5.6). This is well known in the case when $X$ is finite. We will prove the compact case.

First suppose that $G$ is finite. Then, by [22], (5.6.4(c)), we know that $(X, *)=$ $\lim \left(X_{i}, *\right)$ for some system of finite $G$-sets $X_{i}$ such that $G$ acts freely on $\left(X_{i}, *\right)$. Then the result is true by the result in 2.5 about an inverse limit of projectives.

For the general case, for each $N \triangleleft_{o} G, G / N$ acts freely on $(X / N, *)$ so, by the previous paragraph, $R[[X / N, *]]$ is projective over $G / N$. Now apply the result from 2.5 again. 
For the last part, we saw in 2.5 that any projective cover is a product of $P_{S}$ 's, where $S$ is a simple and $P_{S}$ is its projective cover. But $P_{S}$ is a summand of $R[[G]]$.

Lemma 3.12. If $G$ acts freely on $X$ then $F(X)$ is injective and $R[[X]]$ is projective. Conversely any injective in $\mathscr{D}_{R}(G)$ is a summand of some $F(X)$ and any projective in $\mathcal{C}_{R}(G)$ is a summand of some $R[[X]]$, where $G$ acts freely on $X$.

Proof. (Cf. [22], 5.7.1.) The first part follows from 3.11 by adding a disjoint basepoint.

For the second part let $I$ be injective in $\mathscr{D}_{R}(G)$, so its dual $I^{*}$ is profinite. Notice that the action map $G \times I^{*} \rightarrow I^{*}$ induces an equivariant injection $I \cong\left(I^{*}\right)^{*} \cong$ $F\left(I^{*}\right) \rightarrow F\left(G \times I^{*}\right)$, where $G$ acts on $G \times I^{*}$ by left multiplication on the first factor only. This map splits because $I$ is injective.

For any two subgroups $A, B$ of $G$, we write $A \geq_{G} B$ if some $G$-conjugate of $B$ is a subgroup of $A$. We write $A>_{G} B$ if $B$ is conjugate to a proper subgroup of $A$. Since $G$ is compact we can not have $A>_{G} A$.

Define:

$$
\begin{aligned}
X_{\geq H} & =\left\{x \in X \mid \operatorname{Stab}_{G}(x) \geq H\right\}, \quad X_{>H}=\left\{x \in X \mid \operatorname{Stab}_{G}(x)>H\right\}, \\
X_{(\geq H)} & =\left\{x \in X \mid \operatorname{Stab}_{G}(x) \geq_{G} H\right\}, \quad X_{(>H)}=\left\{x \in X \mid \operatorname{Stab}_{G}(x)>_{G} H\right\} .
\end{aligned}
$$

Clearly $X_{\geq H}=X^{H}$ is closed. So is $X_{(\geq H)}$, because it is the image of the map $G \times X_{\geq H} \rightarrow X,(g, x) \mapsto g x$.

Lemma 3.13. $R$ is a continuous $\hat{\mathbb{Z}}_{p}$-module and $F(X, T) \cong F\left(X, \mathbb{Z} / p^{\infty}\right) \check{\bigotimes}_{\hat{\mathbb{Z}}_{p}} T$.

Proof. The first part is clear. Now by duality for $\hat{\mathbb{Z}}_{p}$-modules, $T \cong \mathbb{Z} / p^{\infty} \check{\otimes}_{\hat{\mathbb{Z}}_{p}} T$, thus the natural map from right to left in the statement of the lemma is an isomorphism if $X$ is finite. The general case follows by taking the limit of the finite case.

This means that when dealing with permutation modules we can usually assume that $R=\hat{\mathbb{Z}}_{p}$ and $k=\mathbb{Z} / p$.

Lemma 3.14. Let $X$ be a profinite $G$-set and $Y \subseteq X$ closed. Suppose that $F(X, Y)$ is a summand of a sum of terms $F(G / S)$, where only finitely many different $S$ occur and each $S$ is finite and satisfies $H^{1}(S, R[[Y]])=0$. Then $F(X) \cong F(X, Y) \oplus F(Y)$ in $\mathscr{D}_{R}(G)$.

Proof. There is a short exact sequence $F(X, Y) \rightarrow F(X) \rightarrow F(Y)$; we need to show that it splits in $\mathscr{D}_{R}(G)$. The sequence is classified by an element of $\operatorname{Ext}_{G}^{1}(F(Y), F(X, Y))$; we show that this Ext-group is zero. 
We can write $F(X, Y)$ as a summand of a finite sum of terms $\bigoplus_{\lambda \in \Lambda_{S}} F(G / S)$ for some finite subgroups $S$ and indexing sets $\Lambda_{S}$. Thus $\operatorname{Ext}_{G}^{1}(F(Y), F(X, Y))$ is a summand of a sum of terms $\operatorname{Ext}_{G}^{1}\left(F(Y), \bigoplus_{\lambda \in \Lambda_{S}} F(G / S)\right) \cong \operatorname{Ext}_{G}^{1}(F(Y)$, $\left.\bigoplus_{\lambda \in \Lambda_{S}} \operatorname{Coind}_{S}^{G} T\right) \cong \operatorname{Ext}_{G}^{1}\left(F(Y), \operatorname{Coind}_{S}^{G} \bigoplus_{\lambda \in \Lambda_{S}} T\right) \cong \operatorname{Ext}_{S}^{1}\left(F(Y), \bigoplus_{\lambda \in \Lambda_{S}} T\right)$ (see 2.6).

Since $S$ is finite, $\operatorname{Ext}_{S}^{1}$ agrees with the Ext-group in the category of ordinary $R S$-modules, which we denote by $\operatorname{Ext}_{R S-\text { Mod }}^{1}$. Also $\bigoplus_{\lambda \in \Lambda_{S}} T$ is injective as an $R$ module (e.g. by Baer's Criterion [34], 2.3.1), so it is a summand of $\Pi_{\lambda \in \Lambda_{S}} T$. Hence $\operatorname{Ext}_{R G}^{1}(F(Y), F(X, Y))$ is a summand of a sum of terms $\operatorname{Ext}_{S-\operatorname{Mod}}^{1}\left(F(Y), \Pi_{\lambda \in \Lambda_{S}} T\right) \cong$ $\Pi_{\lambda \in \Lambda_{S}} \operatorname{Ext}_{S-\operatorname{Mod}}^{1}(F(Y), T) \cong \Pi_{\lambda \in \Lambda_{S}} \operatorname{Ext}_{S}^{1}(F(Y), T)$.

It follows from Pontryagin duality that $\operatorname{Ext}_{S}^{1}(F(Y), T)$ is isomorphic to $\operatorname{Ext}_{S}^{1}(R, R[[Y]]) \cong H^{1}(S, R[[Y]])$, which is zero, by hypothesis.

Lemma 3.15. If $G$ is finite and $R$ is torsion free then for any profinite $G$-set $Y$ we have $H^{1}(G, R[[Y]])=0$.

Proof. Since $G$ is finite we can certainly use 2.6 to see that if $Y \cong \lim Y_{i}$ then $H^{1}(G, R[[Y]]) \cong H^{1}\left(G, \lim _{\leftarrow} R\left[Y_{i}\right]\right) \cong \lim H^{1}\left(G, R\left[Y_{i}\right]\right)$.

But $Y_{i}$ is a finite union of $G$-sets of the form $G / H$, so $H^{1}\left(G, R\left[Y_{i}\right]\right)$ is a sum of terms of the form $H^{1}(G, R[G / H]) \cong H^{1}\left(G, \operatorname{Ind}_{H}^{G} R\right) \cong H^{1}(H, R) \cong \operatorname{Hom}(H, R)$, which is 0 because $R$ is torsion free.

Corollary 3.16. Let $X$ be a profinite $G$-set and $Y \subseteq X$ closed. Suppose that $F(X, Y)$ is a summand of a sum of terms $F(G / S)$, where only finitely different $S$ occur and each $S$ is finite. Then $F(X) \cong F(X, Y) \oplus F(Y)$ in $\mathscr{D}_{p}(G)$.

Proof. By 3.13 we may assume that $R$ is $\hat{\mathbb{Z}}_{p}$. Now we can just combine 3.14 and 3.15.

Now we suppose that the action of $G$ on $X$ has only a finite number of conjugacy classes of stabilizers, with representatives $S_{1}, \ldots, S_{n}$. Then each $X_{>S_{i}}$ is closed, because it is the intersection of $X_{\geq S_{i}}$ with the union of the $X_{\left(\geq S_{j}\right)}$ for which $S_{j}>_{G} S_{i}$; hence each $X_{\left(>S_{i}\right)}$ is also closed.

Lemma 3.17. For each $i, F\left(X_{\left(\geq S_{i}\right)}, X_{\left(>S_{i}\right)}\right)$ is a summand of a sum of $F\left(G / S_{i}\right)$ 's.

Proof. The map $\left(G \times_{N_{G}\left(S_{i}\right)} X_{\geq S_{i}}\right) /\left(G \times_{N_{G}\left(S_{i}\right)} X_{>S_{i}}\right) \rightarrow X_{\left(\geq S_{i}\right)} / X_{\left(>S_{i}\right)}$ is a continuous bijection of compact Hausdorff spaces, hence a homeomorphism. Thus

$$
\begin{gathered}
F\left(X_{\left(\geq S_{i}\right)}, X_{\left(>S_{i}\right)}\right) \cong F\left(G \times_{N_{G}\left(S_{i}\right)} X_{\geq S_{i}},\right. \\
\left.G \times_{N_{G}\left(S_{i}\right)} X_{>S_{i}}\right) \cong \operatorname{Coind}_{N_{G}\left(S_{i}\right)}^{G} F\left(X_{\geq S_{i}}, X_{>S_{i}}\right) \cong \operatorname{Coind}_{N_{G}\left(S_{i}\right)}^{G} F\left(X_{\geq S_{i}} / X_{>S_{i}}, *\right) .
\end{gathered}
$$


We have already seen in 3.11 that $F\left(X_{\geq S_{i}} / X_{>S_{i}}, *\right)$ is a summand of a sum of $F\left(N_{G}\left(S_{i}\right) / S_{i}\right)$ 's.

Lemma 3.18. Let $X$ be a profinite $G$-set such that there are only finitely many conjugacy classes of stabilizers and all the stabilizers are finite. Then $F(X) \cong$ $\bigoplus_{i=1}^{n} F\left(X_{\left(\geq S_{i}\right)}, X_{\left(>S_{i}\right)}\right)$.

Proof. Use induction on the number of conjugacy classes of stabilizers that appear. The result is trivial if this number is 0 . Otherwise let $S_{1}$, say, be minimal amongst these stabilizers, and let $Y=\bigcup_{i=2}^{n} X_{\left(\geq S_{i}\right)}$. Now the induction hypothesis shows that $F(Y) \cong \bigoplus_{i=2}^{n} F\left(X_{\left(\geq S_{i}\right)}, X_{\left(>S_{i}\right)}\right)$.

Since $S_{1}$ is minimal, it follows that $X_{\left(\geq S_{1}\right)}$ is open in $X$, because its complement is the union of the $X_{\left(\geq S_{i}\right)}$ where $S_{i}$ contains no conjugate of $S_{1}$ as a subgroup. Thus $F(X, Y) \cong F\left(X_{\left(\geq S_{1}\right)}, X_{\left(>S_{1}\right)}\right)$. Now 3.17 shows that the hypotheses of 3.16 hold, completing the proof.

Lemma 3.19. Discrete R-permutation modules are closed under $\oplus$, hence compact ones are closed under $\Pi$.

Proof. We need to show that the sum of an arbitrary collection of discrete permutation modules is an $R$-permutation module. Let $\left\{X^{s} \mid s \in S\right\}$ be a set of profinite $G$-sets, so $X^{s} \cong \lim _{i \in I_{s}} X_{i}^{s}$, where the $X_{i}^{s}$ are finite $G$-sets.

To each $I_{s}$ add an element $0_{s}$ with the property that $i>0_{s}$ for all $i \in I_{s}$ and call the new directed set $I_{s}^{0}$. Let $X_{0_{s}}^{s}$ be a point $*_{s}$; there is just one map $X_{i}^{s} \rightarrow X_{0_{s}}^{s}$. We still have that $X^{s} \cong \lim _{i \in I_{s}^{0}} X_{i}^{s}$.

For each $s \in S$ choose an element $f(s) \in I_{s}^{0}$ in such a way that for all but finitely many $s \in S$ we have $f(s)=0_{s}$. Consider the infinite sets $Y_{f}=\bigsqcup_{s \in S} X_{f(s)}^{s}$ for all possible choices of $f$. These can be made into an inverse system using the obvious maps. Now, in each $Y_{f}$, identify all the points $*_{s}$ for different $s$ to obtain $\bar{Y}_{f}$. This is consistent with the maps, and the $\bar{Y}_{f}$ form an inverse system of finite $G$-sets. Let $Y=\lim _{Y} \bar{Y}_{f}$.

$Y$ has a fixed point $*$, and it is easy to check that $F(Y, *) \cong \bigoplus_{s \in S} F\left(X^{s}\right)$. Also $F(Y) \cong T \oplus F(Y, *)$, because the inclusion $* \rightarrow Y$ is split by $Y \rightarrow *$.

Definition 3.20. A strict discrete permutation module is a sum of discrete permutation modules of the form $F(G / H)$. A strict compact permutation module is a product of compact permutation modules of the form $R[[G / H]]$. As usual, a strict $R$-permutation module is a summand of a strict permutation module.

Notice that a strict permutation module is an $R$-permutation module by 3.19 . 
Corollary 3.21. If the action of $G$ on $X$ has only a finite number of conjugacy classes of stabilizers and these stabilizers are finite then $R[[X]]$ is a strict $R$-permutation module. If $G$ is finite then any $R$-permutation module is a strict $R$-permutation module.

Lemma 3.22. Any strict $R$-permutation module is a summand of a strict permutation module on a set on which all the stabilizers are pro-p groups.

Proof. This is well known for finite groups. Because we are dealing with strict permutation modules, it suffices to deal with the case of one orbit $G / H$. Let $S \leq H$ be a Sylow pro- $p$ subgroup. We want to show that the natural map $s: R[[G / S]] \rightarrow$ $R[[G / H]]$ splits.

For any open normal subgroup $N \triangleleft_{o} G$ we know, from the finite case, that the quotient map $R[[G / H]] \rightarrow R[G / N H]$ factors through the map $R[G / N S] \rightarrow$ $R[G / N H]$. Let $L_{N}$ be the set of all such factorizations. Then $L_{N}$ is compact, since $R[[G / H]]$ is finitely generated, and the $L_{N}$ form an inverse system. Thus their inverse limit is non-empty, and an element of it is our desired splitting.

Lemma 3.23. Let $U$ and $V$ be compact $R$-permutation modules for a finite group $G$. Then the reduction map $\operatorname{Hom}_{R[G]}(U, V) \rightarrow \operatorname{Hom}_{k[G]}(k \otimes U, k \otimes V)$ is onto.

Proof. By 3.21 we know that $V$ is strict so, because of the way that Hom commutes with products (2.3), we may assume that $U=R[[X]]$ and $V=R[G / H]$. But now $\operatorname{Hom}_{R[G]}(R[[X]], R[G / H]) \cong \operatorname{Hom}_{R[H]}(R[[X]], R)$, so we are reduced to considering the map $\operatorname{Hom}_{R[H]}(R[[X]], R) \rightarrow \operatorname{Hom}_{k[H]}(k[[X]], k)$. But any map $k[[X]] \rightarrow k$ must factor through $k\left[X_{i}\right]$, where $X_{i}$ is some finite quotient of $X$. But $X_{i}$ is a finite union of orbits, so we can reduce to considering the reduction modulo $\mathfrak{m}$ of $\operatorname{Hom}_{R[H]}(R[H / K], R) \cong \operatorname{Hom}_{R[K]}(R, R)$, where our result is obvious.

Lemma 3.24. Let $G$ be finite and $M \in \mathcal{C}_{R}(G)$. Suppose that $M$ is projective over $R$ and that $M^{[P]}=0$ for all non-trivial p-subgroups $P$. Then $M$ is projective.

Proof. It is easy to see, by induction on $|P|$, that $\hat{H}^{0}(P, M)=0$. In particular, this is the case for the Sylow $p$-subgroup $S$, so $M^{[1]}$ is projective over $k S$, by [6], VI 8.5 (see also [4], 2.8), hence $M$ is projective over $R G$.

Now we record some results of Bouc, in the form in which we will need them rather than their most general form. These appeared in [3] for finitely generated modules and [4] for infinitely generated modules.

Theorem 3.25. Let $G$ be a finite group. If $f: L \rightarrow M$ is a morphism between two discrete strict $k$-permutation $k G$-modules such that for every $p$-subgroup $P \leq G$ the 
induced map $L^{[P]} \rightarrow M^{[P]}$ is surjective (resp. injective) then $f$ is a split surjection (resp. split injection).

Proof. See [4], 1.3.

Note that as long as $L$ and $M$ are $k$-permutation modules they are bound to be strict, by 3.21 , so this hypothesis is unnecessary.

Corollary 3.26. In the circumstances of the previous theorem we can make either one of the following alterations.

(1) Each $L_{[P]} \rightarrow M_{[P]}$ is surjective/injective (instead of each $L^{[P]} \rightarrow M^{[P]}$ ).

(2) $L$ and $M$ are compact $k$-permutation modules (rather than discrete).

(3) $L$ and $M$ are compact $R$-permutation modules and each $L^{[P]} \rightarrow M^{[P]}$ is surjective.

Then $f$ is still split.

Proof. Part (1) follows from 3.6; part (2) is its dual.

For part (3) we reduce modulo $\mathfrak{m}$, using 3.5, and obtain a splitting $\bar{s}$ there. By 3.23 we can lift $\bar{s}$ to a map $s: M \rightarrow L$. But $s f$ is the identity modulo $\mathfrak{m}$, so it is surjective, by Nakayama's Lemma. It must split over $R$, so the kernel is a summand, hence 0 , by Nakayama's Lemma again, so $s f$ is an isomorphism. The splitting that we want is $(s f)^{-1} s$.

Recall that a complex is called bounded if only a finite number of the modules are non-zero.

Corollary 3.27. Let $G$ be a finite group.

(1) Let $C$ be a bounded below complex of compact $R$-permutation $R G$-modules such that $C^{[P]}$ is exact for every $p$-subgroup $P$ of $G$ (including the trivial one). Then $C$ is split exact.

(2) Let $C$ be a bounded complex of compact $R$-permutation $R G$-modules. So for some $n \in \mathbb{Z}$ we have $C_{r}=0$ for $r>n$. Suppose that $C^{[P]}$ is exact in degrees $r<n$ for every $p$-subgroup $P$ of $G$. Let $K$ be the kernel of $C_{n} \rightarrow C_{n-1}$. Then $K$ is a summand of $C_{n}$, hence an $R$-permutation module, and the complex $C_{n} / K \rightarrow C_{n-1} \rightarrow \cdots$ is split exact. If, in addition, we assume that $C^{[P]}$ is exact in degree $n$ for all non-trivial p-subgroups $P$ then $K$ is projective.

(3) Let $C$ be a bounded (resp. bounded below) complex of compact R-permutation $R G$-modules such that $C^{[P]}$ is exact for every non-trivial $p$-subgroup $P$ of $G$. Then $C$ is homotopy equivalent to a bounded (resp. bounded below) complex of compact projective $R G$-modules. 
Proof. We prove (1) and (2) by induction on the length of the splitting, i.e. if we let $Z_{m}$ denote the kernel of $C_{m} \rightarrow C_{m-1}$ then we assume that for some $m$ the sequence $Z_{m} \rightarrow C_{m} \rightarrow C_{m-1} \rightarrow \cdots$ is split and use induction on $m$. The assumption implies that $Z_{m}$ is an $R$-permutation module. Since the complex is bounded below, the induction starts.

But each complex $\cdots \rightarrow C_{m+2}^{[P]} \rightarrow C_{m+1}^{[P]} \rightarrow Z_{m}^{[P]}$ also satisfies the hypotheses of the theorem, so, by 3.26, we have a splitting $Z_{m} \rightarrow C_{m+1}$, hence $Z_{m+1} \rightarrow C_{m+1} \rightarrow$ $C_{m} \rightarrow \cdots$ is split.

In case (1) we take the union of the splittings for all $m$.

In case (2) we stop when $m=n$. The additional hypothesis implies that $K$ is projective, by 3.24 .

For (3), let $f: J \rightarrow C$ be a projective resolution of $C$, i.e. $J$ is a bounded below complex of projectives and $f$ is a quasi-isomorphism. Then our assumptions imply that each $f^{[P]}: J^{[P]} \rightarrow C^{[P]}$ is also a quasi-isomorphism. Therefore cone $(f)$ satisfies the conditions of part (1), so cone $(f)$ is split exact and thus $f$ is a homotopy equivalence.

If $C$ is bounded above, let $n$ be such that $C_{r}=0$ for $r \geq n$. Then in degrees greater than or equal to $n$, cone $(f)$ is just $J$ with a degree shift. In particular, $J_{n} \rightarrow J_{n-1}$ is the same as cone $(f)_{n+1} \rightarrow \operatorname{cone}(f)_{n}$, so the kernel $K$ is a summand of $J_{n}$ and hence projective.

Now $K \rightarrow J_{n} \rightarrow J_{n-1} \rightarrow \cdots$ is also a projective cover of $C$, using the restriction of $f$ to $C$ as the map. By the argument above, the map is a homotopy equivalence.

Another approach to permutation modules is taken by Mel'nikov [19] (see also [32]).

\section{Tate hyper-cohomology}

Definition 4.1. If $C$ is a complex of $k G$-modules and $P \leq G$ is a finite $p$-subgroup then let $q: C^{P} \rightarrow C^{[P]}$ be the quotient map. A base map $s: C^{[P]} \rightarrow C^{P}$ is a map of complexes of vector spaces such that the composite $q s: C^{[P]} \rightarrow C^{[P]}$ is homotopic to the identity. We say that $s$ is equivariant if it is a map of complexes of $N_{G}(P) / P$-modules and $q s \simeq$ Id equivariantly.

Remark. If $P$ is a finite $p$-group which acts admissibly on a CW-complex $X$ (i.e. the stabilizer of each cell fixes it pointwise) and $C(X)$ is the CW-chain complex of $X$ over $k$ then the inclusion $X^{P} \subseteq X$ induces an isomorphism $C(X)^{[P]} \cong C\left(X^{P}\right)$ and hence a base map.

Lemma 4.2. Let $P$ be a finite $p$-group and let $C$ be a complex of modules in $\mathcal{C}_{k}(P)$, zero in negative degrees. Let $\tilde{C}$ denote the augmented complex $\cdots \rightarrow C_{1} \rightarrow C_{0} \rightarrow$ 
$H_{0}(C)$. Suppose that both $\tilde{C}^{P}$ and $\tilde{C}^{[P]}$ are exact (e.g. if $\tilde{C}$ is split exact over $k P$ ) and that $q$ induces an isomorphism $q_{-1}: H_{0}(C)^{P} \rightarrow H_{0}(C)^{[P]}$. Then there is a base map $s: C^{[P]} \rightarrow C^{P}$.

If, in addition, $C$ is a complex of modules in $\mathcal{C}_{k}(G)$ and $P<G$ in such a way that $C^{[P]}$ is a complex of projective compact $k\left[\left[N_{G}(P) / P\right]\right]$-modules then the base map can be made equivariant.

Proof. The hypotheses imply that both $C^{P}$ and $C^{[P]}$ are projective resolutions of $H_{0}(C)^{P}$ over $k$. Thus the map $q: C^{P} \rightarrow C^{[P]}$ must be a homotopy equivalence.

In the presence of the extra hypothesis, $C^{[P]}$ is a projective resolution over $k\left[\left[N_{G}(P) / P\right]\right]$. Thus $\left(q_{-1}\right)^{-1}$ lifts to an equivariant chain map $s: C^{[P]} \rightarrow C^{P}$ and $q s \simeq$ Id equivariantly, by an application of the basic comparison theorem ([34], 2.2.6, [6], I 7.4).

We define Tate hyper-cohomology groups over a finite group $G$ in the usual way. If $C$ is a bounded complex (i.e. bounded above and below) of compact $G$-modules then we take a complete resolution $F_{C}$ of $C$ over $G$ by compact modules. We define $\widehat{\mathbb{E x t}}_{G}^{*}(C, D)=H^{*}\left(\operatorname{Hom}_{G}\left(F_{C}, D\right)\right)$ whenever $D$ is a bounded complex of $G$ modules, either compact or discrete. When $C=R$ we write $\hat{H}_{P}^{*}(D)$.

Remark. (a) The complete resolution of a bounded complex $C$ can be formed in the same way as for a module (see [6], VI, X), and has similar uniqueness properties. Take a projective resolution $P$ for $C$ and choose a degree $n$ higher than the upper bound on $C$. Truncate $P$ by removing all terms in degree less than $n$. Now add projectives in all degrees less than $n$ in such a way that the resulting complex is exact, just as in the case of a single module.

If $C$ is projective over $R$ then there is an alternative description of $F_{C}$. Let $F_{R}$ be the complete resolution of the trivial module $R$. Then we can take $F_{C}$ to be $F_{R} \hat{\otimes} C$.

In full generality the complete resolution should be defined as the total complex of the complete version of a Cartan-Eilenberg complex (see [34], 5.7), where all the projective resolutions of modules in the definition are replaced by complete resolutions.

(b) This definition is not quite what we would expect from [6], (VII), which uses the cohomology of $\operatorname{Hom}_{G}\left(F_{R}, \operatorname{Hom}_{R}(C, D)\right)$. But this is isomorphic to $\operatorname{Hom}_{G}\left(F_{R} \hat{\otimes}\right.$ $C, D$ ), and since in [6] $C$ is always projective over $R$ we have that $F_{R} \hat{\otimes} C$ is a complete resolution of $C$. For us such a definition would have the disadvantage that $\operatorname{Hom}_{R}(C, D)$ might only be in $\varepsilon_{R}$ if $D$ is compact.

(c) The construction of complete resolutions via Cartan-Eilenberg resolutions leads formally to two cohomology spectral sequences:

$$
\begin{aligned}
{ }^{\mathrm{I}} E_{1}^{p, q} & =\bigoplus_{p^{\prime}-p^{\prime \prime}}=p \widehat{\operatorname{Ext}}_{G}^{q}\left(C_{p^{\prime}}, D_{p^{\prime \prime}}\right) \Rightarrow \widehat{\mathbb{E x t}}_{G}^{p+q}(C, D), \\
{ }^{\mathrm{II}} E_{2}^{p, q} & =\bigoplus_{q^{\prime}-q^{\prime \prime}=q} \widehat{\operatorname{Ext}}_{G}^{p}\left(H_{q^{\prime}}(C), H_{q^{\prime \prime}}(D)\right) \Rightarrow \widehat{\mathbb{E x t}}_{G}^{p+q}(C, D) .
\end{aligned}
$$


The Cartan-Eilenberg resolutions are themselves double complexes, so the filtration is built in. The case of ordinary hyper-Tor is treated in [34], 5.7, and we can proceed analogously, or simply use the duality of 2.6 to turn our spectral sequence for $\mathbb{E x t}$ into one for Tor.

The boundedness condition on the complexes ensures that there are no convergence problems.

(d) The first spectral sequence shows that $\widehat{\mathbb{E x t}}_{G}^{*}(C, D)$ ignores projective summands of the $C_{p}$, so it vanishes if $C$ is a complex of projectives. The second shows that it is invariant under a quasi-isomorphism of $C$ or $D$.

(e) Since $C$ is quasi-isomorphic to its projective resolution $P$, remark (d) shows that there would be problems associated with allowing unbounded complexes.

The next two lemmas are based on [6], VII 10, [28].

Lemma 4.3. Let $P$ be a cyclic group of order $p$ and let $C$ be a bounded complex of compact permutation $k P$-modules with base map s. Then $\hat{H}_{P}^{*}(C) \cong \hat{H}_{P}^{*}\left(C^{[P]}\right)$.

Proof. Since $C$ consists of permutation modules and both $k$ and $k P$ are indecomposable it follows from 3.21 that, in each degree, $C_{r} \cong(\Pi k) \oplus(\Pi k P)$.

Let cone $(s)$ be the mapping cone of $s: C^{[P]} \rightarrow C$. Consider what happens when we apply the Brauer construction. From the definition, cone $(s)^{[P]}=\operatorname{cone}\left(s^{\prime}\right)$, where $s^{\prime}$ is the induced map $s^{\prime}: C^{[P]} \rightarrow C^{[P]}$. But $s^{\prime}=q s$ is a homotopy equivalence, by the definition of base map, so cone $\left(s^{\prime}\right)$ is exact.

Now 3.27 (3) implies that cone $(s)$ is homotopy equivalent to a bounded complex of projectives. Since $\hat{H}_{P}^{*}$ vanishes on such a complex it follows that $\hat{H}_{P}^{*}(s)$ induces an isomorphism.

Proposition 4.4. Let $P$ be a finite $p$-group and let $C$ be a bounded complex of discrete permutation modules over $k$ for $P$, with a base map for each subgroup of $P$. Then

$$
\sum_{r} \operatorname{dim} H_{r}(C) \geq \sum_{r} \operatorname{dim} H_{r}\left(C^{[P]}\right)
$$

(i.e. if the left hand side is finite then so is the right hand side and the inequality holds).

Proof. Consider a composition series $1=P_{0}<P_{1}<\cdots<P_{n}=P$, where $\left|P_{i}\right|=p^{i}$. Let $s$ be a base map for $C$ as a $P_{i+1}$-module. Then, by 3.5, the composite $\left(C^{\left[P_{i}\right]}\right)^{\left[P_{i+1} / P_{i}\right]} \cong C^{\left[P_{i+1}\right]} \stackrel{s}{\rightarrow} C^{P_{i+1}} \rightarrow C^{P_{i}} \rightarrow C^{\left[P_{i}\right]}$ is a base map for $C^{\left[P_{i}\right]}$ as a $P_{i+1} / P_{i}$-module. Thus we have a proof by induction on $i$ provided that we can prove the case when $|P|=p$.

Note that, since $P$ is cyclic, $\operatorname{dim} \hat{H}_{P}^{r}(M)=\operatorname{dim} \hat{H}^{r}(P, M) \leq \operatorname{dim} M$, for any finite dimensional $k P$-module $M$. 
Consider the spectral sequence

$$
{ }^{\mathrm{II}} E_{2}^{p, q}=\hat{H}_{P}^{p}\left(H_{q}(C)\right) \Rightarrow \hat{H}_{P}^{p+q}(C) .
$$

We see that

$$
\sum_{r} \operatorname{dim} H_{r}(C) \geq \sum_{r} \operatorname{dim} \hat{H}_{P}^{-r}\left(H_{r}(C)\right) \geq \operatorname{dim} \hat{H}_{P}^{0}(C) .
$$

Now, by $4.3, \operatorname{dim} \hat{H}_{P}^{0}(C)=\operatorname{dim} \hat{H}_{P}^{0}\left(C^{[P]}\right)$. Also, by the Künneth Theorem,

$$
\hat{H}_{P}^{0}\left(C^{[P]}\right) \cong \bigoplus_{r} H_{r}\left(C^{[P]}\right) \otimes \hat{H}^{-r}(P),
$$

and $\operatorname{dim} \hat{H}^{-r}(P)=1$ so $\operatorname{dim} \hat{H}_{P}^{0}\left(C^{[P]}\right)=\sum_{r} \operatorname{dim} H_{r}\left(C^{[P]}\right)$.

\section{Weak $\boldsymbol{R}$-permutation resolutions}

Definition 5.1. A compact weak $R$-permutation resolution of the profinite group $G$ is an exact complex of compact $G$-modules

$$
\cdots \rightarrow J_{n} \rightarrow \cdots \rightarrow J_{1} \rightarrow J_{0} \rightarrow R \rightarrow 0 \quad\left(=J_{\bullet}\right),
$$

where each $J_{i}$ is a summand of a permutation module $R\left[\left[X_{i}\right]\right]$ for some profinite space $X_{i}$ on which $G$ acts with finite stabilizers.

The discrete version is just the Pontryagin dual.

A signed weak $R$-permutation resolution is similar except that we allow each $J_{i}$ to be a summand of a product of modules of the form $R\left[\left[X_{i, j}\right]\right] \otimes R^{\prime}$, where $X_{i, j}$ is as before and $R^{\prime}$ is a copy of $R$ on which $G$ acts as \pm 1 .

Remark. Allowing the sign makes no difference to the class of possible modules $J_{i}$ unless char $k=2$ and $2 R \neq 0$, but we want it for the next result.

The next theorem uses a method of Swan [29], following Serre. It was first proved in the profinite case by Scheiderer [25], [24].

Theorem 5.2. If the profinite group $G$ has finite virtual cohomological dimension over $R$ then it has a signed weak $R$-permutation resolution of finite length.

Proof. For some $H \leq_{o} G$ there is a finite projective resolution

$$
0 \rightarrow P_{n} \rightarrow \cdots \rightarrow P_{1} \rightarrow P_{0} \rightarrow R \rightarrow 0
$$

where each $P_{i}$ is of the form $R\left[\left[X_{i}\right]\right]$ for some profinite space $X_{i}$ on which $H$ acts freely (using the Eilenberg swindle). 
Take $J_{\bullet}$ to be the tensor induced complex, as defined in [1] or [29]. Note that there is a rather subtle sign convention. The terms are all summands of $R\left[\left[\left(\sqcup_{\pi} \Pi_{i=1}^{s} g_{i} X_{\pi_{i}}\right]\right]\right.$, where $\left\{g_{1}, \ldots, g_{s}\right\}$ are coset representatives of $G / H$ and $\pi:\{1, \ldots, s\} \rightarrow\{1, \ldots, n\}$, except that they might be twisted by a sign.

Lemma 5.3. If $H<G$ is closed and $M$ is a discrete $G$-module and $N$ a compact $G$-module then $\operatorname{Ext}_{H}^{*}(N, M)=\underline{\lim }_{H<K<_{0} G} \operatorname{Ext}_{K}^{*}(N, M)$ (the limit over restriction maps).

Proof.

$$
\begin{aligned}
& \operatorname{Ext}_{H}^{*}(N, M) \cong \operatorname{Ext}_{G}^{*}\left(N, \operatorname{Coind}_{H}^{G} \operatorname{Res}_{H}^{G} M\right) \\
& \cong \operatorname{Ext}_{G}^{*}(N, F(G / H) \ddot{\otimes} M) \text { by } 2.4 \\
& \cong \operatorname{Ext}_{G}^{*}\left(N,\left(\lim _{H<K<<_{0} G} F(G / K)\right) \check{\otimes} M\right) \\
& \cong \operatorname{Ext}_{G}^{*}\left(N, \lim _{H<K<_{o} G}(F(G / K) \check{\otimes} M)\right) \\
& \cong \lim _{H<K<_{o} G} \operatorname{Ext}_{G}^{*}(N, F(G / K) \check{\otimes} M) \\
& \cong \lim _{\longrightarrow<K<<_{o} G} \operatorname{Ext}_{K}^{*}(N, M) \text {. }
\end{aligned}
$$

Proposition 5.4. Let $G$ act on the profinite space $X$ and let $M$ be a discrete $G$ module and $N$ a compact $G$-module. Suppose that, for some $n$ and every $x \in X$, $\operatorname{Ext}_{\operatorname{Stab}_{G}(x)}^{n}(N, M)=0$. Then $\operatorname{Ext}_{G}^{n}\left(N \hat{\otimes}_{R} R[[X]], M\right)=0$.

Similarly, if $X$ is based and $\operatorname{Ext}_{\operatorname{Stab}_{G}(x)}^{n}(N, M)=0$ for every $x \in X \backslash\{*\}$ then $\operatorname{Ext}_{G}^{n}\left(N \hat{\otimes}_{R} R[[X, *]], M\right)=0$.

Proof. The unbased result follows from the based one by adding a disjoint basepoint, so we prove the based statement.

Let $X=\lim X_{i}$, where $i$ runs through some inverse system $I$, each $X_{i}$ is a finite $G$-set, and for $\overleftarrow{j} \geq i \in I$ let $\rho_{i, j}$ denote the map $X_{j} \rightarrow X_{i}$ and $\rho_{j}$ the map $X \rightarrow X_{j}$. Then $\operatorname{Ext}_{G}^{n}(N \hat{\otimes} R[[X, *]], M) \cong \operatorname{Ext}_{G}^{n}\left(N \hat{\otimes} \lim _{\leftarrow}^{R}\left[\left[X_{i}, *\right]\right], M\right) \cong \operatorname{Ext}_{G}^{n}\left(\lim _{\leftarrow}(N \hat{\otimes}\right.$ $\left.\left.R\left[\left[X_{i}, *\right]\right]\right), M\right) \cong \lim _{\longrightarrow} \operatorname{Ext}_{G}^{n}\left(N \hat{\otimes} R\left[\left[X_{i}, *\right]\right], \overleftarrow{M}\right)$.

For $H<_{o} G \overrightarrow{\text { we }}$ use the Eckmann-Shapiro isomorphism in order to obtain $\operatorname{Ext}_{G}^{n}(N \hat{\otimes} \quad R[G / H], M) \cong \operatorname{Ext}_{G}^{n}\left(\operatorname{Ind}_{H}^{G} \operatorname{Res}_{H}^{G} N, M\right) \cong \operatorname{Ext}_{H}^{n}(N, M) \cong$ $\left(\bigoplus_{x \in G / H} \operatorname{Ext}_{\operatorname{Stab}_{G}(x)}^{n}(N, M)\right)^{G}$, where the map from the left hand side to the right hand side is induced by restriction to $\operatorname{Stab}_{G}(x)$ and then inclusion of the summand $N \otimes x \subset N \otimes R[G / H]$.

Thus $\lim _{\longrightarrow} \operatorname{Ext}_{G}^{n}\left(N \hat{\otimes} R\left[\left[X_{i}, *\right]\right], M\right) \cong \lim _{\longrightarrow}\left(\left(\bigoplus_{x \in X_{i} \backslash\{*\}} \operatorname{Ext}_{\operatorname{Stab}_{G}(x)}^{n}(N, M)\right)^{G}\right) \cong$ $\left(\lim _{\rightarrow} \bigoplus_{x \in X_{i} \backslash\{*\}} \operatorname{Ext}_{\operatorname{Stab}_{G}(x)}^{n}(N, M)\right)^{G}$. We will describe explicitly the maps in the limit system in the last term (before taking fixed points). 
Let $A_{i}=\bigoplus_{x \in X_{i} \backslash\{*\}} \operatorname{Ext}_{\operatorname{Stab}_{G}(x)}^{n}(N, M)$ and $x \in X_{i} \backslash\{*\}$, and let moreover $a \in \operatorname{Ext}_{\mathrm{Stab}_{G}(x)}^{n}(N, M)$ be regarded as an element of $A_{i}$. Then for any $j>i$ the image of $a$ in $A_{j}$ has component at $y \in X_{j}$ equal to 0 if $\rho_{i, j}(y) \neq x$ and equal to $\operatorname{res}_{\mathrm{Stab}_{G}(y)}^{\mathrm{Stab}_{G}(x)} a$ if $\rho_{i, j}(y)=x$.

We need to show that, for every $i \in I, x \in X_{i}$ and $t \in \operatorname{Ext}_{\operatorname{Stab}_{G}(x)}^{n}(N, M)$ there is a $j>i$ such that if $z \in X_{j}$ with $\rho_{i, j}(z)=x$ then $\operatorname{res}_{\operatorname{Stab}(z)}^{\operatorname{Stab}(z)} t=0$.

It is helpful if, given $i, x$, , we let $H=\operatorname{Stab}_{G}(x)$ and $Y_{j}=\left(\rho_{i, j}\right)^{-1}(x)$, for $j \geq i$. Then the $Y_{j}$ are $H$-sets and $\left(\rho_{i}\right)^{-1}(x) \cong \lim _{j \geq i} Y_{j}$ is a profinite $H$-set. We need to show that there is a $k \geq i$ such that if $z \in Y_{k}$ then $\operatorname{res}_{\operatorname{Stab}_{H}(z)}^{H} t=0$.

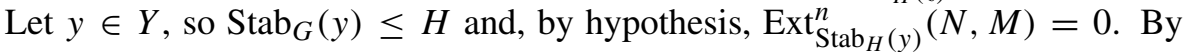
5.3 there is an open subgroup $H_{y}<_{o} H$ such that $\operatorname{Stab}_{H}(y) \leq H_{y}$ and $\operatorname{res}_{H_{y}}^{H} t=0$. Now there must be a $j_{y} \geq i$ such that $\operatorname{Stab}_{H}\left(\rho_{j_{y}}(y)\right) \leq H_{y}$.

Let $U_{y}=\left(\rho_{j_{y}}\right)^{-1}\left(\rho_{j_{y}}(y)\right) \subseteq Y$. Then $\left\{U_{y} \mid y \in Y\right\}$ is an open covering of $Y$. Since $Y$ is compact we can let $\left\{U_{y_{1}}, \ldots, U_{y_{r}}\right\}$ be a finite subcover and let $k \in I$ be such that $k \geq j_{y_{i}}, i=1, \ldots, r$.

Now if $z \in Y_{k}$ we have $z=\rho_{k}(\tilde{z})$ for some $\tilde{z} \in Y$, so $\tilde{z} \in U_{y_{m}}$ for some $m$ and thus $\operatorname{Stab}_{H}\left(\rho_{y_{m}}(\tilde{z})\right) \leq H_{y_{m}}$. But $\rho_{y_{m}}(\tilde{z})=\rho_{k, y_{m}}(z)$ so $\operatorname{Stab}_{H}(z) \leq H_{y_{m}}$ and $\operatorname{res}_{\mathrm{Stab}_{H}(z)}^{H} t=0$.

Corollary 5.5. If $G$ has a signed weak $R$-permutation resolution of finite length $n$, and $M \in \mathcal{C}_{R}(G)$ is projective on restriction to each finite elementary abelian subgroup, then $M$ has projective dimension less than or equal to $n$ in $\mathcal{C}_{R}(G)$.

There is a dual result for discrete modules.

Proof. By Chouinard's Theorem (see e.g. [1], Vol. II, 5.2.4), $M$ is projective on restriction to any finite subgroup. In particular, it is projective over $R$ so we can tensor the resolution

$$
0 \rightarrow J_{n} \rightarrow \cdots \rightarrow J_{1} \rightarrow J_{0} \rightarrow R \rightarrow 0
$$

with $M$ over $R$ to obtain

$$
0 \rightarrow J_{n} \hat{\otimes} M \rightarrow \cdots \rightarrow J_{1} \hat{\otimes} M \rightarrow J_{0} \hat{\otimes} M \rightarrow M \rightarrow 0 .
$$

By 5.4, each $J_{i} \hat{\otimes} M$ satisfies $\operatorname{Ext}_{G}^{*}\left(J_{i} \hat{\otimes} M, N\right)=0$ for all discrete modules $N$ and so, by 2.5 , is projective.

The next useful lemma is taken from [2].

Lemma 5.6. Let $H<_{o} G$ and suppose that $M$ is a compact module for $G$ of finite projective dimension whose restriction to $H$ is projective. Then $M$ is projective. 
Proof. Let $Y$ be the cokernel of the co-augmentation $R \rightarrow R[G / H]$. We obtain a short exact sequence

$$
0 \rightarrow M \rightarrow R[G / H] \hat{\otimes} M \rightarrow Y \hat{\otimes} M \rightarrow 0 .
$$

The middle term is projective by 2.5 , and if $M$ has projective dimension $r$ then we can take a projective resolution of length $r$ and tensor it with $Y$ to see that $Y \hat{\otimes} M$ has projective dimension at most $r$. It follows that either $M$ has projective dimension at most $r-1$, a contradiction, or that $M$ is projective.

Lemma 5.5 and the theory of profinite duality groups discussed in [33] are precisely what is needed to carry over the algebraic proof of the local cohomology theorem of Benson and Greenlees [2] from discrete virtual duality groups to profinite ones. In particular we obtain the following result.

Theorem 5.7. If $G$ is a virtual duality group of virtual dimension $n$ and with dualizing module $I$, then for any compact $G$-module $M$ there is a spectral sequence

$$
E_{2}^{* * *}=H_{J}^{* * *}\left(H^{*}(G, M)\right) \Rightarrow \Sigma^{n} H_{*}(G, I \hat{\otimes} M),
$$

where $J$ is the ideal of positive degree elements in $H^{*}(G)$.

\section{6. $R$-permutation resolutions}

In this section we consider algebraic analogues of some geometric results of Kropholler and Mislin [17].

We continue to suppose that char $k=p>0$ and we count the trivial group as a finite $p$-subgroup. Recall that the Brauer quotient at the trivial group is taken to mean reduction modulo $\mathfrak{m}$.

Definition 6.1. By an $R$-permutation resolution for $G$ of we mean a complex of compact $G$-modules

$$
\cdots \rightarrow C_{r} \rightarrow \cdots \rightarrow C_{1} \rightarrow C_{0} \rightarrow R \quad(=\tilde{C}),
$$

where each $C_{i}$ is an $R$-permutation module with finite stabilizers.

In addition, if we apply the Brauer quotient at any finite $p$-subgroup $P \leq G$, the resulting complex $\tilde{C}^{[P]}$ is required to be exact.

We say that the $R$-permutation resolution is of finite type if each $C_{i}$ is finitely generated. It is of length $n$ if $C_{n} \neq 0$ but $C_{r}=0$ for $r>n$. It is strict if all the $C_{r}$ are strict. 
We adopt the convention that $C$ denotes the unaugmented complex and $\tilde{C}$ the augmented one.

Definition 6.2. The smallest possible length of a strict $R$-permutation resolution for $G$ is called the permutation dimension of $G$ over $R$ (possibly $\infty$ ), which we abbreviate to permdim $G$.

Notice that if $H<G$ then the restriction of an $R$-permutation resolution or a signed weak permutation resolution for $G$ to $H$ is still a resolution of the same sort for $H$. However it might no longer be of finite type or strict, even if it was before.

A resolution of finite type is necessarily strict, by 3.10 .

Lemma 6.3. If $C$ is an R-permutation resolution for $G$ and $P<G$ is a finite $P$ subgroup then $C^{[P]}$ is a k-permutation resolution for $N_{G}(P) / P$. If $C$ is strict or of finite type then so is $C^{[P]}$.

Proof. The first part is by 3.4. The second by 3.7 and 3.10 .

We have given the definition of an $R$-permutation resolution in terms of the Brauer construction, because this is what corresponds better with the geometric methods used for discrete groups. However, there is an alternative description, by a theorem of Bouc mentioned previously (3.27).

Proposition 6.4. The condition that the resolution be exact at every Brauer quotient at a finite p-subgroup is equivalent to the condition that it be split exact on restriction to every finite p-subgroup (or equivalently on restriction to every finite subgroup).

Remark. Let $\mathcal{F}$ denote the class of strict $R$-permutation modules with finite stabilizers in $\mathcal{C}_{R}(G)$. Then, in the language of relative homological algebra, a strict $R$-permutation resolution for $G$ is the same thing as a right $\mathcal{F}$-resolution of $R$ in $\mathcal{C}_{R}(G)$ ([11]) or a projective resolution of $R$ for the class $\mathcal{F}$ ([10]).

An obvious analogue for $\mathcal{C}_{R}(G)$ of the comparison theorem in relative homological algebra now tells us that if a strict $R$-permutation resolution for $G$ exists then it is unique up to homotopy.

The usual proof that such resolutions always exist only transfers to $\mathcal{C}_{R}(G)$ if $G$ has only a finite number of conjugacy classes of finite $p$-subgroups, otherwise we run into a problem with direct sums (cf. the proof of 6.6).

The relative Ext groups are defined by setting $\operatorname{Ext}_{G, \mathcal{F}}^{*}(R, N)$ to be the homology of $\operatorname{Hom}_{G}(C, N)$, where $C$ is a strict $R$-permutation resolution for $G$. Thus permdim $G$ can be characterized as the largest degree in which not all of these groups vanish. 
Definition 6.5. The relative dimension of $G$ over $R, \operatorname{reldim}_{R} G$, is the least integer $n$ such that every compact module for $G$ that is projective on restriction to every finite $p$-subgroup has projective dimension less than or equal to $n$. If no such $n$ exists then we set $\operatorname{reldim}_{R} G=\infty$.

If $M \in \mathcal{C}_{R}(G)$ is projective on restriction to every finite $P$-subgroup then the same is true of $k \otimes_{R} M \in \mathcal{C}_{k}(G)$. Also if $M \in \mathcal{C}_{R}(G)$ is projective over $R$ then its projective dimension is the same as that of $k \otimes_{R} M \in \mathcal{C}_{k}(G)$. It follows that $\operatorname{reldim}_{R} G \leq \operatorname{reldim}_{k} G$.

We saw in 5.5 that $\operatorname{reldim}_{k} G \leq \operatorname{permdim} G$.

Recall that a module $M$ is said to be of type $\mathrm{FP}_{n}$ if there is a projective resolution $\cdots \rightarrow P_{1} \rightarrow P_{0} \rightarrow M$ in which $P_{i}$ is finitely generated for $i \leq n$ (see [6], VIII 4.3).

Proposition 6.6. Let $G$ be a profinite group with only a finite number of conjugacy classes of finite p-subgroups $P$, and let $N \in \mathcal{C}_{R}(G)$. Then there is a short exact sequence $L \rightarrow M \stackrel{f}{\rightarrow} N$ such that $M$ is a strict $R$-permutation module $M$ with finite stabilizers and $f^{[P]}: M^{[P]} \rightarrow N^{[P]}$ is surjective for every finite $p$-subgroup $P$. This can be done in such a way as to satisfy the following properties.

(1) If, for some $p$-subgroup $P$, we have $N^{[Q]}=0$ for all finite $p$-subgroups $Q>P$ then also $M^{[Q]}=0$ for all $Q>P$ and $f^{[P]}: M^{[P]} \rightarrow N^{[P]}$ is a projective cover of $k\left[\left[N_{G}(P) / P\right]\right]$-modules. Also $N^{[P]}$ is projective on restriction to any $Q / P$ for $P \triangleleft Q$.

(2) If $N$ becomes $R$-permutation on restriction to some $P$ then $f$ is split over $P$, so $L$ is $R$-permutation on restriction to $P$ and there is a short exact sequence $L^{[P]} \rightarrow M^{[P]} \rightarrow N^{[P]}$.

(3) If each $N^{[P]}$ is finitely generated over $k\left[\left[N_{G}(P) / P\right]\right]$ then $M$ is finitely generated.

(4) If each $N^{[P]}$ is of type $\mathrm{FP}_{n}$ over $k\left[\left[N_{G}(P) / P\right]\right]$ for some $n \geq 1$ then each $\operatorname{ker}\left(f^{[P]}: M^{[P]} \rightarrow N^{[P]}\right)$ is of type $\mathrm{FP}_{n-1}$. If, in addition, we assume that $N$ is $R$-permutation on restriction to each $P$ then $L^{[P]}$ is of type $\mathrm{FP}_{n-1}$.

Proof. For each finite $p$-subgroup $P$, let $K_{P} \stackrel{i}{\rightarrow} N^{[P]}$ be the projective cover of $N^{[P]}$ as an $R\left[\left[N_{G}(P) / P\right]\right]$-module, which we inflate to an $R\left[\left[N_{G}(P)\right]\right]$-module. Lift $i$ to $K_{P} \stackrel{\phi}{\rightarrow} N^{P}$ and let $\operatorname{Ind}_{N_{G}(P)}^{G} K_{P} \stackrel{\tilde{\phi}}{\rightarrow} N$ be the adjoint map.

Let $M=\Pi_{P \in \mathcal{P} / G} \operatorname{Ind}_{N_{G}(P)}^{G} K_{P}$ and let $f$ be the product of the $\tilde{\phi}_{P}$ 's. This is permissible because $\mathcal{P} / G$ is finite. Then $L=\operatorname{ker}(f)$ and the surjectivity condition is guaranteed by Lemma 6.8 below.

For (1), the first two conditions are consequences of lemmas 6.7 and 6.8 below. The last is because the hypothesis implies that $\hat{H}^{0}\left(Q / P, N^{[P]}\right)=0$ so the claim follows by 3.24 . 
(2) follows from 3.26 and (3) is by construction.

For (4), notice that, since $M$ is a finitely generated $R$-permutation module with finite stabilizers, so is each $M^{[P]}$, by 3.7. Since $G$ contains only a finite number of conjugacy classes of finite $p$-groups, there is an open normal $p$-torsion-free subgroup $U$. Given $P$, let $H$ be the image of $U \cap N_{G}(P)$ in $N_{G}(P) / P$, which is still an open subgroup.

An $N_{G}(P) / P$-module is of type $\mathrm{FP}_{r}$ if and only if its restriction to $H$ is of type $\mathrm{FP}_{r}$ (cf. [6], VIII 5.1). On restriction to $H, M^{[P]}$ is finitely generated and projective. Thus $\operatorname{ker}\left(f^{[P]}\right)$ is of type $\mathrm{FP}_{n-1}$ over $H$, and hence over $N_{G}(P) / P$, by Schanuel's Lemma (see [6], VIII 4.3).

We still need to prove the two lemmas, so let $P$ be a finite $p$-subgroup of $G$ and $K$ a projective module in $\mathcal{C}_{R}\left(N_{G}(P) / P\right)$, which we inflate to an $N_{G}(P)$-module.

Lemma 6.7. If $Q$ is another p-subgroup of $G$ that is not conjugate to a subgroup of $P$ then $\left(\operatorname{Ind}_{N_{G}(P)}^{G} K\right)^{[Q]}=0$.

Proof. It suffices to treat the case when $K=R\left[\left[N_{G}(P) / P\right]\right]$, so $\operatorname{Ind}_{N_{G}(P)}^{G} K \cong$ $R[[G / P]]$. But, by $3.4, R[[G / P]]^{[Q]} \cong k\left[\left[(G / P)^{Q}\right]\right]=0$.

Continuing with the same notation, suppose that $N$ is a $G$-module and that there is an $N_{G}(P)$-module map $i: K \rightarrow N^{[P]}$. Suppose that $i$ factors as $K \stackrel{\phi}{\rightarrow} N^{P} \stackrel{q}{\rightarrow} N^{[P]}$, where $q$ is the quotient map. Let $\operatorname{Ind}_{N_{G}(P)}^{G} K \stackrel{\tilde{\phi}}{\rightarrow} N$ be the adjoint map to $\phi$.

Lemma 6.8. The map $\tilde{\phi}^{[P]}:\left(\operatorname{Ind}_{N_{G}(P)}^{G} K\right)^{[P]} \rightarrow N^{[P]}$ has the same image as $i$, and $\left(\operatorname{Ind}_{N_{G}(P)}^{G} K\right)^{[P]} \cong k \otimes_{R} K$.

Proof. First we treat the case when $K=R[[X]]$, where $X$ is a profinite $N_{G}(P) / P$ set with a free action. Then $\operatorname{Ind}_{N_{G}(P)}^{G} K \cong R\left[\left[G \times_{N_{G}(P)} X\right]\right]$. The inclusion of $X$ in $G \times_{N_{G}(P)} X$ as $N_{G}(P) \times_{N_{G}(P)} X$ yields an isomorphism $j: k[[X]] \rightarrow R\left[\left[G \times_{N_{G}(P)}\right.\right.$ $X]]^{[P]} \cong\left(k[[G]] \hat{\otimes}_{k\left[\left[N_{G}(P)\right]\right]} k[[X]]\right)^{[P]}$, and $\tilde{\phi}^{[P]} j=i$.

There is a map $\operatorname{End}_{k\left[\left[N_{G}(P) / P\right]\right]}(k[[X]]) \rightarrow \operatorname{End}_{k[[G]]}\left(k[[G]] \hat{\otimes}_{k\left[\left[N_{G}(P)\right]\right]} k[[X]]\right)$ given by $f \mapsto \operatorname{Id} \hat{\otimes} f$, and this is compatible with $j$.

In the general case, $K$ is a summand of such an $R[[X]]$ and the isomorphism $j$ is compatible with the idempotent splitting off $K$.

Theorem 6.9. Suppose that there are only finitely many conjugacy classes of finite p-subgroups $P$ of $G$. Then the following are equivalent.

(1) Each $N_{G}(P) / P$ has a signed weak k-permutation resolution of finite length.

(2) $G$ has a strict R-permutation resolution of finite length. 
(3) Each reldim $_{k} N_{G}(P) / P$ is finite.

Proof. (2) implies (1) by 6.3 and (1) implies (3) by 5.5, so we assume that each $\operatorname{reldim}_{k} N_{G}(P) / P$ is finite and construct a strict $R$-permutation complex for $G$.

In fact, starting with any module $N \in \mathcal{C}_{R}(G)$ that is an $R$-permutation module on restriction to each finite $p$-subgroup, we will construct a complex with all the same properties except that the last term is $N$ instead of $R$.

Define $r(N)$ to be the smallest $r$ for which $N^{[P]}=0$ for all finite $p$-subgroups $P$ with $|P| \geq p^{r}$. The size of the finite $p$-subgroups is bounded, so $r$ exists.

Use 6.6 repeatedly to produce a resolution $\cdots \rightarrow C_{r} \rightarrow \cdots \rightarrow C_{1} \rightarrow C_{0} \rightarrow N$ $(=\tilde{C})$ by $R$-permutation modules with finite stabilizers. In fact, in the notation of 6.6, if $N$ is $R$-permutation with finite stabilizers on restriction to any finite subgroup then so is $L$ and $r(L) \leq r(N)$. Each $\tilde{C}^{[P]}$ is exact, by 6.6(2), so the problem is to show $\tilde{C}$ is of finite length.

If $r(N)=0$ then $N^{[1]}=0$, so $N=0$.

If $r(N) \geq 1$ then for any $P$ of order $p^{r(N)-1}, 6.6(1)$ shows that $\tilde{C}^{[P]}$ is the minimal projective resolution of $N^{[P]}$ in $\mathcal{C}_{k}\left(N_{G}(P) / P\right)$. The last part of 6.6(1) also shows that $N^{[P]}$ is projective on restriction to any finite $p$-subgroup, so our assumption that $\operatorname{reldim}_{k} N_{G}(P) / P$ is finite implies that the resolution $\tilde{C}^{[P]}$ stops.

Since there are only finitely many subgroups of order $p^{r(N)-1}$ up to conjugacy, if we progress far enough along the resolution then $N^{\prime}=\operatorname{ker}\left(C_{n} \rightarrow C_{n-1}\right)$ will satisfy $r\left(N^{\prime}\right)<r(N)$. But $\cdots \rightarrow C_{n+2} \rightarrow C_{n+1} \rightarrow N^{\prime}$ is the resolution for $N^{\prime}$, so it must stop, by induction.

Lemma 6.10. Let $G$ be a group of finite virtual cohomological dimension t over $R$ and let $P$ be a finite $p$-subgroup of $G$. Then $N_{G}(P) / P$ has finite virtual cohomological dimension less than or equal to $t$.

Proof. Let $U<G$ be open and of cohomological dimension $t$. Then $H=U \cap N_{G}(P)$ is open in $N_{G}(P)$ and has cohomological dimension less than or equal to $t$. But $H$ can contain no $p$-torsion, so it is isomorphic to its image in $N_{G}(P) / P$.

The construction in the next proof is based on one introduced by Samy-Modeliar [23] for finite $p$-groups.

Proposition 6.11. Let $G$ be a profinite group of finite virtual cohomological dimension such that its Sylow pro-p-subgroup has only a finite number of conjugacy classes of finite p-subgroups $P$, and let $N \in \mathcal{C}_{R}(G)$. Assume that $N$ is $R$-permutation on restriction to each $P$ and that each $N^{[P]}$ is projective on restriction to some open subgroup of $N_{G}(P) / P$. Then $N$ is an R-permutation module with finite stabilizers. 
Proof. Let $S$ denote the Sylow pro- $p$-subgroup of $G$. From 3.22 we know that $R$ is a summand of $R[[G / S]]$, so $N$ is a summand of $R[[G / S]] \hat{\otimes} N \cong \operatorname{Ind}_{S}^{G} \operatorname{Res}_{S}^{G} N$, thus it is enough to prove the result for $N$ restricted to $S$, and we will assume that $G$ is a pro- $p$-group.

We follow the proof of 6.6 , but we construct $M$ in a more economical way. By downward induction on $r$ we will construct an $R$-permutation module with finite stabilizers $M_{r}$, which is in fact a product of modules $R[[G / P]]$ for finite $p$-subgroups $P$ with $|P| \geq p^{r}$, and a map $f_{r}: M_{r} \rightarrow N$ such that $f_{r}^{[P]}: M_{r}^{[P]} \rightarrow N^{[P]}$ is an isomorphism for all finite $p$-subgroups $P$ with $|P| \geq p^{r}$.

For large enough $r$ we can take $M_{r}=0$, so the induction starts. Suppose that we have $f_{r+1}$ and we want to construct $f_{r}$.

Let $P$ be a subgroup of order $p^{r}$ and consider $f_{r+1}^{[P]}: M_{r+1}^{[P]} \rightarrow N^{[P]}$. Let $q: N^{[P]} \rightarrow N^{[P]} / \operatorname{Im}\left(f_{r+1}^{[P]}\right)$ be the quotient map and let $\pi: K_{P} \rightarrow N^{[P]} / \operatorname{Im}\left(f_{r+1}^{[P]}\right)$ be a projective cover in $\mathcal{C}_{R}\left(N_{G}(P) / P\right)$. Lift this to a map $i: K_{P} \rightarrow N^{[P]}$, which factors through a map $\bar{K}_{P}=k \otimes K_{P} \rightarrow N^{[P]}$, and combine the latter with $f_{r+1}^{[P]}$ to obtain a surjection $g: \bar{K}_{P} \oplus M_{r+1}^{[P]} \rightarrow N^{[P]}$. We claim that $g$ is an isomorphism.

In order to prove this, let $L_{P}$ be the kernel of $g$, so we have a short exact sequence $L_{P} \rightarrow \bar{K}_{P} \oplus M_{r+1}^{[P]} \rightarrow N^{[P]}$ in $\mathcal{C}_{k}\left(N_{G}(P) / P\right)$. If we restrict to any $p$-subgroup $Q / P$ then this is split and $L_{P}$ is projective over $Q / P$, by 3.27.

By hypothesis, $N^{[P]}$ is projective over some open subgroup $H<N_{G}(P) / P$. Also $\bar{K}_{P}$ and $M_{r+1}^{[P]}$ are projective over $H$, by construction, so $L_{P}$ is projective over $H$. It follows from 5.5, using 5.2, 5.5 and 6.10, that $L_{P}$ is projective over $N_{G}(P) / P$.

Thus $L_{P}$ is injective relative to $H$ and, since the short exact sequence is split over $H$, it is also split over $N_{G}(P) / P$.

Let $V$ be an indecomposable factor of $L_{P}$ and let $\alpha: V \rightarrow \bar{K}_{P} \oplus M_{r+1}^{[P]}$ be the inclusion map and $\beta$ a splitting. Let $e_{K}$ and $e_{M}$ be the idempotent endomorphisms of $\bar{K}_{P} \oplus M_{r+1}^{[P]}$ corresponding to the summands. So $\operatorname{Id}_{V}=\beta \alpha=\beta e_{K} \alpha+\beta e_{M} \alpha$. Since $\operatorname{End}(V)$ is local, at least one of $\beta e_{K} \alpha$ and $\beta e_{M} \alpha$ must be an automorphism.

If it is $\beta e_{K} \alpha$ then $e_{K} \alpha(V)$ is a summand of $\bar{K}_{P}$. But $0=q g \alpha=q g e_{K} \alpha+$ $q g e_{M} \alpha=q i e_{K} \alpha+q f_{r+1}^{[P]} e_{M} \alpha=\pi e_{K} \alpha$. This contradicts the definition of $\pi$ as a projective cover. Therefore $e_{M} \alpha(V)$ is a summand of $M_{r+1}^{[P]}$. This is not possible either, by Lemma 6.12 below.

We have proved that $g$ is an isomorphism, (so, in fact, $N^{[P]} / \operatorname{Im}\left(f_{r+1}^{[P]}\right)$ was projective and $q$ was split from the beginning).

Now consider $K_{P}$ as an $N_{G}(P) / P$-module and lift $i$ to a map $\phi_{P}: K_{P} \rightarrow N^{P}$. Let $\tilde{\phi}_{P}: \operatorname{Ind}_{N_{G}(P)}^{G} K_{P} \rightarrow N$ be the adjoint map. Let $M_{r}=M_{r+1} \oplus \Pi_{P \in \mathcal{P}_{r} / G} \operatorname{Ind}_{N_{G}(P)}^{G} K_{P}$, where $\mathcal{P}_{r}$ is the set of $p$-subgroups of order $p^{r}$. There is an obvious map $f_{r}: M_{r} \rightarrow N$ with components $f_{r+1}$ and the $\tilde{\phi}_{P}$ 's. This $f_{r}$ induces an isomorphism $M_{r}^{[P]} \rightarrow N^{[P]}$ when $|P| \geq p^{r}$. 
This completes the induction, so we have a map $f_{0}: M_{0} \rightarrow N$ that is an isomorphism modulo $\mathfrak{m}$, hence an isomorphism.

This construction of $M_{r}$ can be used, instead of the one in 6.6, in the proof of 6.9, to produce a smaller resolution.

Lemma 6.12. Let $G$ be a pro- $p$ group and $P$ a finite subgroup of order $p^{r}$. Suppose that $M \in \mathcal{C}_{R}(G)$ is a product of $R[[G / Q]]$ 's for finite subgroups $Q$ with $|Q|>p^{r}$. Then $M^{[P]}$, considered as a module for $N_{G}(P) / P$, does not contain a projective summand.

Proof. Since we are dealing with pro- $p$ groups, each $R\left[\left[\left(N_{G}(P) / P\right) / S\right]\right]$ is indecomposable. This is because there is only one simple module $k$ and $\operatorname{Hom}\left(R\left[\left[\left(N_{G}(P) / P\right) / S\right]\right], k\right)$ has dimension 1 .

In particular, the only indecomposable projective is the free module of rank 1, so, to obtain a contradiction, assume that $R\left[\left[N_{G}(P) / P\right]\right]$ is isomorphic to a summand of a product of $R[[G / Q]]^{[P]}$ 's.

We have a surjection $\prod R\left[\left[G / Q_{\lambda}\right]\right]^{[P]} \rightarrow R\left[\left[N_{G}(P) / P\right]\right]$. Compose this with the augmentation $R\left[\left[N_{G}(P) / P\right]\right] \rightarrow k$. Since $k$ is finite and the maps are continuous, the composition factors through a finite product. But now one of this finite set of factors must map onto $k$, and, by Nakayama's Lemma, it maps onto $R\left[\left[N_{G}(P) / P\right]\right]$. The latter is projective, so the map is split, and $R\left[\left[N_{G}(P) / P\right]\right]$ is a summand of some $R[[G / Q]]^{[P]} \cong R\left[\left[(G / Q)^{P}\right]\right]$.

But, by 3.7, $\operatorname{Stab}_{N_{G}(P) / P}(g Q)=\left(N_{G}(P) \cap{ }^{g} Q\right) / P$, where $P<{ }^{g} Q$. Since $Q$ is a $p$-group, $N_{g} Q(P)$ is strictly bigger than $P$, so the stabilizer is non-trivial. Thus $R\left[\left[(G / Q)^{P}\right]\right]$ is a finite sum of terms $R\left[\left[\left(N_{G}(P) / P\right) / S\right]\right]$ for some non-trivial $S \leq N_{G}(P) / P$, and each of these is indecomposable. This contradicts the assumption that $R\left[\left[N_{G}(P) / P\right]\right]$ is a summand.

Corollary 6.13. Suppose that there is a bound on the number of conjugacy classes of finite p-subgroups of a Sylow p-subgroup of $G$. If $G$ has virtual cohomological dimension $t$ over $R$ then permdim $G=\operatorname{reldim}_{k} G=\operatorname{reldim}_{R} G=t$.

Proof. Let $\cdots \rightarrow C_{r} \rightarrow \cdots \rightarrow C_{1} \rightarrow C_{0} \rightarrow R(=\tilde{C})$ be any strict permutation resolution. Let $N=\operatorname{ker}\left(C_{t-1} \rightarrow C_{t-2}\right)$.

For each $P$ we know that $N$ is $R$-permutation on restriction to $P$. Also $N_{G}(P) / P$ contains an open subgroup $H$ of cohomological dimension less than or equal to $t$, by 6.10; if we restrict $\tilde{C}^{[P]}$ to $H$ we see that $N$ is the $t$ th syzygy of $k$, so is projective.

Now 6.11 shows that $N$ is an $R$-permutation module with finite stabilizers, so we can finish the resolution with $0 \rightarrow N \rightarrow C_{t-1} \rightarrow C_{t-2} \rightarrow \cdots$.

Thus permdim $G \leq t$, and $\operatorname{reldim}_{k} G \leq \operatorname{permdim} G$ by 5.5 . Also $\operatorname{reldim}_{R} G \leq$ $\operatorname{reldim}_{k} G$, by the remark after 6.5 . Finally, it is easy to see that $t \leq \operatorname{reldim}_{R} G$, by considering $\operatorname{Ind}_{H}^{G} R$, where $H \triangleleft_{o} G$ is of cohomological dimension $t$. 
Example. Let $p=3$ and $G=P S L_{2}\left(\hat{\mathbb{Z}}_{3}\right)$. The structure of this group is well known (see [15] for example), but the reader can also consider this example as applying to any group with the following properties. $G$ is virtually pro-3, and (by the theory of Lazard [18]), vcd $G=3$. It is easy to verify that there is only one non-trivial finite 3subgroup up to conjugacy, $Q \cong \mathbb{Z} / 3$ (generated by $\left(\begin{array}{cc}0 & 1 \\ -1 & -1\end{array}\right)$ ), and $N_{G}(Q) \cong Q \times \hat{\mathbb{Z}}_{3}$. By 5.2 and 5.5, $G$ satisfies condition (3) of 6.9.

We apply the construction of 6.6. $R^{[Q]} \cong k$ and its projective cover over $N_{G}(Q) / Q \cong \hat{\mathbb{Z}}_{p}$ is $\operatorname{Ind}_{1}^{N_{G}(Q) / Q} R \cong R\left[\left[N_{G}(Q) / Q\right]\right]$, which we inflate to an $N_{G}(Q)$ module. Also $R^{[1]} \cong k$ and we denote the projective cover of this by $P_{k}$. We could in fact use any projective module that maps onto $k$, such as a free module. So our resolution starts $\cdots \rightarrow P_{k} \oplus R\left[\left[N_{G}(Q) / Q\right]\right] \rightarrow R$. Let $N$ be the kernel.

Now $N^{[Q]}=0$, so $N$ is projective on restriction to $Q$. Since $\operatorname{reldim}_{k} G$ is finite, $N$ has a finite projective resolution $C_{r} \rightarrow \cdots \rightarrow C_{1} \rightarrow N$ (any one will do), and we have an $R$-permutation resolution $C_{r} \rightarrow \cdots \rightarrow C_{1} \rightarrow P_{k} \oplus R\left[\left[N_{G}(Q) / Q\right]\right] \rightarrow R$.

Let $K=\operatorname{ker}\left(C_{2} \rightarrow C_{1}\right)$. Let $H$ be an open subgroup of finite cohomological dimension. Over $H$, the module $K$ is a third syzygy, so must be projective. It follows from 5.6 that $K$ is projective over $G$.

So we have an $R$-permutation resolution

$$
K \rightarrow C_{2} \rightarrow C_{1} \rightarrow P_{k} \oplus R\left[\left[N_{G}(Q) / Q\right]\right] \rightarrow R .
$$

If we use the construction of 6.11 instead, the only significant difference is that we do not use $P_{k}$ at the first stage, so we obtain a resolution of the form $K^{\prime} \rightarrow C_{2}^{\prime} \rightarrow$ $C_{1}^{\prime} \rightarrow R\left[\left[N_{G}(Q) / Q\right]\right] \rightarrow R$.

Recall that a group $G$ is said to be of type FP (over $R$ ) if there is a projective resolution of $R$ in $\mathcal{C}_{R}(G)$ of finite type and finite length. It is of type $v$ FP if it possesses an open subgroup of type FP. $G$ is of type $\mathrm{FP}_{\infty}$ if there is a projective resolution of $R$ of finite type.

Theorem 6.14. The following are equivalent.

(1) G has a strict R-permutation resolution of finite type and finite length.

(2) $G$ has only finitely many conjugacy classes of finite p-subgroups $P$ and each $N_{G}(P) / P$ (including $G$ ) is virtually of type FP.

(3) There are only finitely many conjugacy classes of finite p-subgroups $P$ and each $N_{G}(P) / P$ (including $G$ ) is of type $F P_{\infty}$ and $\operatorname{reldim}_{k} N_{G}(P) / P<\infty$.

Proof. (1) $\Rightarrow(2)$ : Let $C$ be a strict $R$-permutation resolution for $G$ of finite type and finite length and let $P<G$ be a finite $p$-subgroup. Exactness of $\tilde{C}^{[P]}$ implies that $C_{0}^{[P]} \neq 0$. But if $C_{0}$ is a summand of $\bigoplus_{i=1}^{n} R\left[\left[G / S_{i}\right]\right]$ then $P$ must be conjugate to 
a subgroup of some $S_{i}$. Thus there are only a finite number of conjugacy classes of finite $p$-subgroups.

Now we can find an open subgroup $H \leq G$ that avoids all the finite $p$-subgroups. The restriction of the resolution to $H$ is then a projective resolution, and still of finite type, so $G$ is virtually of type FP.

Now $C^{[P]}$ is a strict $k$-permutation resolution for $N_{G}(P) / P$ of finite type and finite length by 6.3 , so the same argument shows that $N_{G}(P) / P$ is virtually of type FP.

(2) $\Rightarrow$ (3): This follows the fact that type $v$ FP implies type $\mathrm{FP}_{\infty}$ ([6], VIII 5.1) and from 5.2 and 5.5.

(3) $\Rightarrow(1)$ : The construction of Theorem 6.9 yields a complex of finite type at each step by 6.6 parts (3) and (4).

Let $H$ be a profinite group, $Q$ a finite group and $X$ a finite $Q$-set. We write $H$ wr $_{X} Q$ for the wreath product $H^{X} \rtimes Q$, where the commutation relation is given by $\left(q f q^{-1}\right)(x)=f\left(q^{-1} x\right), q \in Q, f \in H^{X}, x \in X$.

If $S_{n}$ denotes the symmetric group on the set $\underline{\mathrm{n}}=\{1, \ldots, n\}$ then $H$ \& $S_{n}=$ $H \operatorname{wr}_{\mathrm{n}} S_{n}$.

Lemma 6.15. If $H$ is p-torsion free then $H \geq S_{n}$ has only finitely many conjugacy classes of finite p-subgroups.

Proof. Any finite $p$-subgroup maps isomorphically to its image in $S_{n}$, so we only need to show that any two finite $p$-subgroups with the same image, $P$ say, in $S_{n}$ are conjugate. This is equivalent to showing that all sections of $H \mathrm{wr}_{\mathrm{n}} P \rightarrow P$ are conjugate, which in turn is equivalent to showing that the non-abelian cohomology group $H^{1}\left(P, H^{\mathrm{n}}\right)$ is trivial (see [27], I 5).

But $H^{1}\left(P, H^{X \sqcup Y}\right) \cong H^{1}\left(P, H^{X}\right) \times H^{1}\left(P, H^{Y}\right)$, so we can reduce to the case of a transitive $P$-set $P / Q$. But then $H^{1}\left(P, H^{P / Q}\right) \cong H^{1}(Q, H) \cong \operatorname{Hom}(Q, H)$, by [27], I 5.7, so is trivial.

Corollary 6.16. If $H$ is of finite cohomological dimension over $R$ then $H$ i $S_{n}$ has a strict $R$-permutation resolution of finite length.

Proof. $H_{2} S_{n}$ is of finite virtual cohomological dimension, so by 6.10 so is each $N_{G}(P) / P$ for each finite $p$-subgroup $P$. In view of 6.15 we can apply 6.9 ; condition (1) is satisfied, by 6.10 , and condition (2) is our desired conclusion.

Corollary 6.17. If $G$ is of finite virtual cohomological dimension then it has an $R$-permutation resolution of finite length.

Proof. Let $H<G$ be open of finite cohomological dimension and set $n=|G / H|$. Then $H$ < $S_{n}$ has an $R$-permutation resolution of finite length, by 6.16. But $G$ embeds in $H$ ? $S_{n}$, so we can restrict this resolution to $G$. 
From now on we write $H^{*}(G)$ for $H^{*}(G, k)$.

Proposition 6.18. If $G$ has an open normal subgroup of finite cohomological dimension and with finite dimensional homology (i.e. finite dimensional in each degree and zero in large degrees), then there are only finitely many conjugacy classes of finite p-subgroups $P$, and each $N_{G}(P)$ has an open normal subgroup of finite cohomological dimension and with finite dimensional homology. If $G$ is virtually pro- $p$ then this subgroup may be taken to be pro- $p$.

Proof. (Cf. [6], IX 13.2.) Let $H \triangleleft_{o} G$ be of finite cohomological dimension and with finite homology, hence $p$-torsion free ( $H$ can be chosen to be pro- $p$ if $G$ is virtually pro- $p$ ). Let $X$ be a compact $R$-permutation resolution of $G$ of finite length, which exists by 6.17. The restriction of $X$ to $H$ is a projective resolution of $R$. Let $Y=k \otimes_{H} X$, so that $H_{*}(Y) \cong H_{*}(H)$, by the definition of homology, and so $H_{*}(Y)$ is finite.

Let $P$ be a $p$-subgroup of $G / H$. We claim that $Y$ has a base map over $P$. Recall from 3.8 that $\varsigma_{H}(P)$ is the set of finite $p$-subgroups of $G$ that map isomorphically to $P<G / H$, and $H$ acts on this by conjugation. Assume for the moment that $\varsigma_{H}(P) / H$ is finite.

Observe that each module in $X$ is a summand of a module of the form $R[[V]]$, where $V$ is a profinite $G$-set with finite stabilizers. Now 3.8 becomes $(V / H)^{P} \cong$ $\amalg_{Q \in S_{H}(P) / H} V^{Q} / N_{H}(Q)$, and this is a homeomorphism because the union is finite. The dual of 3.9 is thus

$$
R[[V / H]]^{[P]} \cong \bigoplus_{Q \in \$_{H}(P) / H} k \otimes_{N_{H}(Q)} R[[V]]^{[Q]}
$$

and so

$$
Y^{[P]} \cong \bigoplus_{Q \in S_{H}(P) / H} k \otimes_{N_{H}(Q)} X^{[Q]} .
$$

Now $X^{[Q]}$ is a complex of projectives over $N_{H}(Q) / Q$, so each $X^{Q} \rightarrow X^{[Q]}$ has an $N_{H}(Q) / Q$-equivariant base map, by 4.2 , and these piece together to give a base map

$$
\begin{aligned}
Y^{[P]} \cong \bigoplus_{Q \in \delta_{H}(P) / H} k \otimes_{N_{H}(Q)} X^{[Q]} & \rightarrow \bigoplus_{Q \in \delta_{H}(P) / H} k \otimes_{N_{H}(Q)} X^{Q} \\
& \rightarrow\left(k \otimes_{H} X\right)^{P}=Y .
\end{aligned}
$$

It follows that $H_{*}\left(Y^{[P]}\right)$ is finite, by 4.4.

Now from the identity $\dagger$ and the fact that $X^{[Q]}$ is a projective resolution of $N_{H}(Q) / Q$ we see that

$$
H_{*}\left(Y^{[P]}\right) \cong \bigoplus_{Q \in S_{H}(P) / H} H_{*}\left(N_{H}(Q)\right) .
$$

It follows that for each $Q, H_{*}\left(N_{H}(Q)\right)$ is finite. 
It remains to prove our assertion that $\delta_{H}(P) / H$ is finite. Our argument is based on the one in [20], but is technically more elementary (see the remark before 6.20). Notice that this claim for all $P$ of order $p^{r}$ is equivalent to the claim that $G$ has only finitely many conjugacy classes of finite $p$-subgroups of that order. The proof is by induction on the order of $P$. We know that $H^{*}(G)$ is finitely generated by a spectral sequence argument and the theory of Evens [12], [13], [1], so a theorem of Quillen [21] implies that the number of conjugacy classes of elementary abelian $p$-subgroups is finite (see also [20]).

Otherwise $P$, of order $p^{r}$, contains a central cyclic subgroup $C$ of order $p$, and $P \leq N_{G}(C)$. We may conjugate in such a way that $C$ is one of a given finite set of representatives. It now suffices to show that there are only finitely many conjugacy classes of subgroups of $N_{G}(C)$ of order $p^{r}$ containing $C$ or, equivalently, that there are only finitely many conjugacy classes of subgroups of $N_{G}(C) / C$ of order $p^{r-1}$.

Since $\varsigma_{H}(C)$ is finite, we can certainly apply the argument above with $C$ in place of $P$. We find that $N_{H}(C) \unlhd_{o} N_{G}(C)$ is open normal of finite cohomological dimension and with finite homology, and this injects into $N_{G}(C) / C$. Our induction hypothesis now applies to $N_{G}(C) / C$ and we are done.

Theorem 6.19. If $G$ is a virtual pro- $p$ group then the following conditions are equivalent.

(1) $G$ has a strict $R$-permutation resolution of finite type and finite length.

(2) $G$ is virtually of type FP.

(3) There are only finitely many conjugacy classes of finite p-subgroups $P$ and $\operatorname{reldim}_{k} N_{G}(P) / P<\infty$ and for each simple $R\left[\left[N_{G}(P) / P\right]\right]$-module $S$ each $H^{i}\left(N_{G}(P) / P, S\right)$ is of finite dimension.

(4) For each simple $R[[G]]$-module $S$ its cohomology $H^{*}(G, S)$ is noetherian as a module over $H^{*}(G)$.

Proof. We claim that conditions (1),(2) and (3) are equivalent to those of 6.14 under the hypothesis that $G$ is a virtual pro- $p$-group.

Clearly (1) is the same in both. Also the present (2) is equivalent to 6.14 (2) using 6.18 , because if $H$ is a torsion free pro- $p$ subgroup of $N_{G}(P)$ with finite dimensional homology then it injects into $N_{G}(P) / P$, and for a pro- $p$ group finite homology implies type FP.

The two conditions (3) are equivalent, because the $H^{i}\left(N_{G}(P) / P, S\right)$ measure the growth of the minimal projective resolution ([33], 4.2.3).

Now (2) implies (4), by considering the Lyndon-Hochschild-Serre spectral sequence. The converse is shown for pro- $p$ groups in [20]. In fact, the method of that paper shows that there is an open pro- $p$ subgroup $K<_{o} G$ such that res: $H^{*}(G) \rightarrow$ $H^{*}(K)$ is zero in positive degrees. This can be seen by using 5.3 with $H=1$. Let 
$x_{1}, \ldots, x_{m}$ generate $H^{*}(G)$ as a $k$-algebra. For each $x_{i}$ there is an open subgroup $K_{i}<_{o} G$ such that $\operatorname{res}_{K_{i}}^{G} x_{i}=0$. Let $K=\cap_{i} K_{i}$. Since $H^{*}(K) \cong H^{*}\left(G, \operatorname{Coind}_{K}^{G} k\right)$ and $\operatorname{Coind}_{K}^{G} k$ has a finite filtration by simple modules it follows that $H^{*}(K)$ is finitely generated over $H^{*}(G)$. Since the $x_{i}$ act as 0 , we see that $H^{*}(K)$ must be finite-dimensional and $K$ must be of type FP.

Remark. The deeper part of [20] shows that if $G$ is a pro- $p$ group and $H^{*}(G)$ is finitely generated then the number of conjugacy classes of finite $p$-subgroups of $G$ is finite. It uses results of Dwyer and Wilkerson [9], and also work of Lannes on unstable algebras over the Steenrod algebra, to obtain a version of 6.20 below (see also [15]). The method of the present paper bypasses that.

We simplify the statement of the next result by restricting ourselves to pro- $p$ groups.

Theorem 6.20. Let $G$ be a pro-p group with finitely generated cohomology as a ring. Then there are only finitely many conjugacy classes of finite p-subgroups $P$, and for each one $H^{*}\left(N_{G}(P) / P\right)$ is finitely generated as a ring and $H^{*}\left(N_{G}(P)\right)$ is finitely generated as a module over $H^{*}(G)$.

Proof. The first claim is just part of 6.19. For the second assertion we use 6.19 to change the question to one about the existence of a strict $k$-permutation resolution of finite type and finite length. But this is inherited by $N_{G}(P) / P$, by 6.3 .

For the third claim, let $U \triangleleft_{o} G$ be pro- $p$ of type FP (using 6.19 condition (2)). Now $N_{G}(P) / P$ is virtually of type FP, by the first claim and 6.19 again. It follows that the image of $U \cap N_{G}(P)$ in $N_{G}(P) / P$ is of type $\mathrm{FP}$, and this is isomorphic to $U \cap N_{G}(P)$.

Consider the diagram

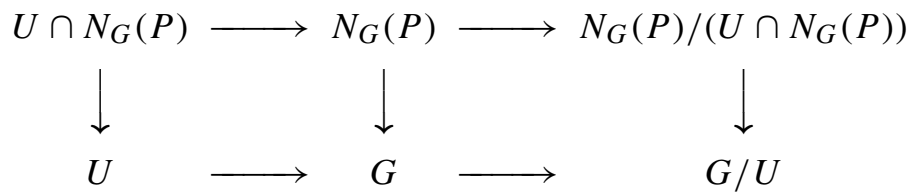

The Lyndon-Hochschild-Serre spectral sequence for the top row has $E_{2}$ page $H^{*}\left(N_{G}(P) /\left(U \cap N_{G}(P)\right), H^{*}\left(U \cap N_{G}(P)\right)\right)$. Via inflation this is noetherian over $H^{*}\left(N_{G}(P) /\left(U \cap N_{G}(P)\right)\right)$, hence so is $H^{*}\left(N_{G}(P)\right)$, by the theory of Evens [12], $[13,1]$.

By the same theory, $H^{*}\left(N_{G}(P) /\left(U \cap N_{G}(P)\right)\right)$ is noetherian over $H^{*}(G / U)$ via restriction (we are dealing with finite groups here).

Combining these, we find that $H^{*}\left(N_{G}(P)\right)$ is noetherian over $H^{*}(G / U)$ via the natural map $N_{G}(P) \rightarrow G / U$. But this map factors through $G$, so $H^{*}\left(N_{G}(P)\right)$ must be noetherian over $H^{*}(G)$. 


\section{Tate-Farrell cohomology}

For any group of finite virtual cohomological dimension we can construct a complete resolution as in [6], X, and so deal with the Tate-Farrell cohomology or hypercohomology, just as we did for the Tate cohomology of a finite group in $\S 4$. This was first considered for profinite groups by Scheiderer [25], but the approach given here is more elementary and allows compact coefficients, as required in certain applications, [30] and [31] for example.

This cohomology has the expected properties, as in [6]. In particular it can be calculated using subgroup complexes, but in order to state this we need to define the chain complex of the Quillen complex.

For any group $U$, let $\Delta(U)$ denote the usual Quillen complex for $U$, with $n$ simplices corresponding to the elements of $\Delta_{n}(U)$, the chains of elementary abelian $p$-subgroups of $U$. For $U$ finite and $X$ a finite poset with an action of $U$, let $T \rightarrow$ $D_{0} \rightarrow \cdots \rightarrow D_{r}(=\tilde{D}(|X|))$ denote the complex of cochains on $|X|$ with coefficients in $T$, and $C_{r} \rightarrow \cdots \rightarrow C_{0} \rightarrow R(=\tilde{C}(|X|))$ the dual complex of chains over $R$. These are complexes of permutation modules over $U$.

For $N \triangleleft_{o} G$ p-torsion free, let $\Delta_{n}^{N}(G)$ denote the image of $\Delta_{n}(G)$ in $\Delta_{n}(G / N)$. If $M<N$ there are natural maps $\Delta_{n}^{M}(G) \rightarrow \Delta_{n}^{N}(G)$. Notice that $\Delta_{n}(G) \cong$ $\lim _{n} \Delta_{n}^{N}(G)$ as sets, and in this way we give $\Delta_{n}(G)$ a profinite topology.

Define $\tilde{D}(\Delta(G))=\lim _{\longrightarrow} \tilde{D}\left(\Delta^{N}(G)\right)$ and $\tilde{C}(\Delta(G))=\lim _{\leftarrow} \tilde{C}\left(\Delta^{N}(G)\right)$. Thus $D_{n}(\Delta(G)) \cong \lim _{\longrightarrow} F\left(\Delta_{n}^{N}(\overrightarrow{G)})\right.$.

Lemma 7.1. For any non-trivial finite $p$ subgroup $P \leq G$ both $\tilde{D}(\Delta(G))_{[P]}$ and $\tilde{C}(\Delta(G))^{[P]}$ are exact.

Proof. Denote by $\Delta_{n}^{N, P}(G)$ the image of $\Delta_{n}(G)^{P}$ in $\Delta_{n}^{N}(G)$, so that we have $\Delta_{n}(G)^{P} \cong \lim _{\leftarrow} \Delta_{n}^{N, P}(G)$.

The simplicial complex $\Delta^{N, P}(G)$ is contractible via the usual poset maps $\bar{E} \geq \overline{E^{P}} \leq \overline{E^{P} Z_{p}(P)} \geq \overline{Z_{p}(P)}$, where bars denote images in $G / N, E$ is an elementary abelian $p$-subgroup of $G$ invariant under conjugation by $P$ and $Z_{p}(G)$ is the maximal central elementary abelian $p$-subgroup of $P$. Notice that $E^{P} Z_{p}(P)$ is actually an elementary abelian subgroup of $G$, and if $E$ and $E^{\prime}$ have the same image in $G / N$ then so do $E^{P} Z_{p}(P)$ and $E^{\prime P} Z_{p}(P)$. Thus $\tilde{D}\left(\Delta^{N, P}(G)\right)$ is exact.

Now $F\left(\Delta_{n}(G)\right)_{[P]} \cong F\left(\Delta_{n}(G)^{P}\right) \cong F\left(\lim \Delta_{n}^{N, P}(G)\right) \cong \lim _{\longrightarrow} F\left(\Delta_{n}^{N, P}(G)\right)$, so $\tilde{D}(\Delta(G))_{[P]} \cong \lim _{\longrightarrow} \tilde{D}\left(\Delta^{N, P}(G)\right)$ is exact.

The compact case is dual.

Lemma 7.2. If there are only finitely many conjugacy classes of elementary abelian p-subgroups then $D_{n}(\Delta(G)) \cong \bigoplus_{\sigma \in \Delta(G)_{n} / G} F\left(G / \operatorname{Stab}_{G}(\sigma)\right)$ and $C_{n}(\Delta(G)) \cong$ $\bigoplus_{\sigma \in \Delta(G)_{n} / G} R\left[\left[G / \operatorname{Stab}_{G}(\sigma)\right]\right]$. 
Proof. $D_{n}(\Delta(G))=\lim _{\longrightarrow} D_{n}\left(\Delta^{N}(G)\right) \cong \lim _{\longrightarrow} F\left(\Delta_{n}^{N}(G)\right) \cong F\left(\lim _{\longleftarrow} \Delta_{n}^{N}(G)\right) \cong$ $F\left(\Delta_{n}(G)\right)$ and $F\left(\Delta_{n}(\overrightarrow{G))}\right.$ has the form claimed by 3.10 .

The compact case is dual.

Remark. In [20] it is shown that a pro- $p$ group has a finite number of conjugacy classes of elementary abelian $p$-subgroups if and only if its cohomology is finitely generated as a ring modulo nilpotent elements.

Let $C(\Delta(G))$ denote the complex $\tilde{C}(\Delta(G))$, but without the augmentation. By analogy with the case of discrete groups we expect the following result.

Theorem 7.3. Let $M$ be a $G$-module, discrete or compact. Then

$$
\widehat{\mathbb{E x t}}_{G}^{*}(C(\Delta(G)), M) \cong \hat{H}^{*}(G, M) .
$$

Proof. From the long exact sequence associated to $C \rightarrow \tilde{C} \rightarrow R$ we see that it suffices to show that $\widetilde{\mathbb{E x t}}_{G}^{*}(\tilde{C}(\Delta(G)), M)=0$.

By induction on $r$ we can add projectives to $\tilde{C}(\Delta(G))$ in degrees less than or equal to $r$ in such a way that the resulting complex $\tilde{C}^{r}(\Delta(G))$ is exact in degrees strictly less than $r$. Let $d$ be the length of the longest chain of elementary abelian $p$-subgroups and consider $\tilde{C}^{d}(\Delta(G))$. This is zero in degrees greater than $d$ and has homology only in degree $d$; call this homology group $K$.

By $3.27(2), K$ is projective over any finite $p$-subgroup, so, by 5.2 and $5.5, K$ has finite projective dimension, $e$ say, over $G$.

Now consider $\tilde{C}^{d+e}(\Delta(G))$. It has only one homology group, $L$ say, in degree $d+e$. But $L$ is the $e$ th syzygy of $K$, so is projective. Let $\bar{C}(\Delta(G))$ be the complex obtained from $\tilde{C}^{d+e}(\Delta(G))$ by adding $L$ in degree $d+e+1$, with the obvious boundary map. Then $\bar{C}(\Delta(G))$ is exact.

But adding projective modules to a complex does not effect $\mathbb{E x t}$, since it need not change the complete resolution, so $\widehat{\mathbb{E x t}}_{G}^{*}(\tilde{C}(\Delta(G)), M) \cong \widehat{\mathbb{E x t}}_{G}^{*}(\bar{C}(\Delta(G)), M)$, and a complete resolution for $\bar{C}(\Delta(G))$ is just 0 , so $\widehat{\mathbb{E x t}}_{G}^{*}(\bar{C}(\Delta(G)), M)=0$.

Remark. $\widehat{\mathbb{E x t}}_{G}^{*}(C(\Delta(G)), M)$ can be computed using the spectral sequence

$$
{ }^{\mathrm{I}} E_{1}^{p, q}=\widehat{\operatorname{Ext}}_{G}^{q}\left(C(\Delta(G))_{p}, M\right) \Rightarrow \widehat{\mathbb{E x t}}_{G}^{p+q}(C(\Delta(G)), M) .
$$

If there are only a finite number of conjugacy classes of elementary abelian $p$ subgroups then, by $7.2,{ }^{\mathrm{I}} E_{1}^{p, q} \cong \bigoplus_{\sigma \in \Delta_{p}(G) / G} \hat{H}^{q}\left(\operatorname{Stab}_{G}(\tilde{\sigma}), M\right)$, (where $\tilde{\sigma}$ denotes a chain in the class $\sigma$ ).

This is known to be a powerful tool for calculation in the case of discrete groups. In the context of profinite groups, the case of $p$-rank 1 and coefficients $k$ already appears in the work of Henn [15], where it is used to make calculations. 


\section{References}

[1] D. J. Benson, Representations and Cohomology I; II. Cambridge Stud. Adv. Math. 30; 31, Cambridge University Press, Cambridge 1992. Zbl 0908.20001 MR 1644252; Zbl 0908.20002 MR 1634407

[2] D. J. Benson and J. P. C. Greenlees, Commutative algebra for cohomology rings of virtual duality groups. J. Algebra 192 (1997), 678-700. Zbl 0901.20040 MR 1452682

[3] S. Bouc, Résolutions de foncteurs de Mackey. In Group representations: cohomology, group actions and topology (Seattle, WA, 1996), Proc. Sympos. Pure Math. 63, Amer. Math. Soc., Providence, RI, 1998, 31-83. Zbl 0897.19001 MR 1603131

[4] S. Bouc, Le complexe de chaînes d'un G-complexe acyclique. J. Algebra 220 (2000), 415-436. Zbl 0940.55020 MR 1717349

[5] M. Broué, On Scott modules and $p$-permutation modules: an approach through the Brauer morphism. Proc. Amer. Math. Soc. 93 (1985), 401-408. Zbl 0574.20005 MR 0773988

[6] K. S. Brown, Cohomology of Groups. Grad. Texts in Math. 87, Springer-Verlag, Berlin 1982. Zbl 0584.20036 MR 0672956

[7] A. Brumer, Pseudocompact algebras, profinite groups and class formations. J. Algebra 4 (1966), 442-470. Zbl 0146.04702 MR 0202790

[8] A. Douady, Cohomologie des groupes compacts totalement discontinus (d'après Tate). Séminaire Bourbaki 1959/1960, exposé 189, Soc. Math. France, Paris 1995, 287-298. Zbl 0149.27701 MR 1603474

[9] W. G. Dwyer and C. Wilkerson, Smith Theory and the functor T. Comment. Math. Helv. 66 (1991), 1-17. Zbl 0726.55011 MR 1090162

[10] S. Eilenberg, and J. C. Moore, Foundations of Relative Homological Algebra. Mem. Amer. Math. Soc. 55 (1965). Zbl 0129.01101 MR 0178036

[11] E. E. Enochs and O. M. G. Jenda, Relative Homological Algebra. De Gruyter Exp. Math. 30, Walter de Gruyter, Berlin 2000. Zbl 0952.13001 MR 1753146

[12] L. Evens, The cohomology ring of a finite group. Trans. Amer. Math. Soc. 101 (1961), 224-239. Zbl 0104.25101 MR 0137742

[13] L. Evens, The cohomology of groups. Oxford Math. Monogr., Oxford University Press, New York 1991.Zbl 0742.20050 MR 1144017

[14] P. Gabriel, Des catégories abéliennes. Bull. Soc. Math. France 90 (1962), 323-448. Zbl 0201.35602 MR 0232821

[15] H. W. Henn, Centralizers of elementary abelian $p$-groups and mod- $p$ cohomology of profinite groups. Duke Math. J. 91 (1998), 561-585. Zbl 0948.20027 MR 1604171

[16] I. Kaplansky, Infinite Abelian groups. University of Michigan Press, Ann Arbor, MI, 1954. Zbl 0057.01901 MR 0065561

[17] P. H. Kropholler and G. Mislin, Groups acting on finite dimensional spaces with finite stabilizers. Comment. Math. Helv. 73 (1998), 122-136. Zbl 0927.20033 MR 1610595

[18] M. Lazard, Groupes analytiques p-adiques. Inst. Hautes Études Sci. Publ. Math. 26 (1965), 369-603. Zbl 0139.02302 MR 0209286 
Vol. 82 (2007)

[19] O. V. Mel'nikov, Aspherical pro-p-groups. Mat. Sb. 193 (2002), 71-104. Zbl 1064.20030 MR 1937030

[20] P. A. Minh and P. Symonds, Cohomology and finite subgroups of profinite groups. Proc. Amer. Math. Soc. 132 (2004), 1581-1588. Zbl 1048.20012 MR 2051117

[21] D. Quillen, The spectrum of an equivariant cohomology ring II. Ann. of Math. 94 (1971), 573-602. Zbl 0247.57013 MR 0298694

[22] L. Ribes and P.Zalesskii, Profinite Groups. Ergeb. Math. Grenzgeb. (3) 40, Springer-Verlag, Berlin 2000. Zbl 0949.20017 MR 1775104

[23] Samy-Modeliar, M., Certaines constructions liées aux foncteurs de Mackey cohomologiques. Doctoral thesis, Université de Paris VI, 2005.

[24] C. Scheiderer, Real and Étale cohomology. Lecture Notes in Math. 1588, Springer-Verlag, Berlin 1991. Zbl 0852.14003 MR 1321819

[25] C. Scheiderer, Farrell cohomology and Brown theorems for profinite groups. Manuscripta Math. 91 (1996), 247-281. Zbl 0885.20017 MR 1411658

[26] J. P. Serre, Sur la dimension cohomologique des groupes profinis. Topology 3 (1965), 413-420. Zbl 0136.27402 MR 0180619

[27] J. P. Serre, Galois Cohomology. 5th edition, Lecture Notes in Math. 5, Springer-Verlag, Berlin 1997. Zbl 0902.12004 MR 1466966

[28] R. G. Swan, A new method in fixed point theory. Comment. Math. Helv. 34 (1960), 1-16. Zbl 0144.22602 MR 0115176

[29] R. G. Swan, Groups of cohomological dimension one. J. Algebra 12 (1969), 585-601. Zbl 0188.07001 MR 0240177

[30] P. Symonds, The Tate-Farrell cohomology of the Morava stabilizer group $S_{p-1}$ with coefficients in $E_{p-1}$. In Homotopy theory: relations with algebraic geometry, group cohomology, and algebraic K-theory, Contemp. Math. 346, Amer. Math. Soc., Providence, RI, 2004, 485-492. Zbl 1069.55010 MR 2066512

[31] P. Symonds, On the construction of permutation complexes for profinite groups. Geom. Topol., to appear.

[32] P. Symonds, Double coset formulas for profinite groups. Preprint.

[33] P. Symonds and T. Weigel, Cohomology of p-adic analytic groups. In New Horizons in pro-p groups (ed. by M. du Sautoy, D. Segal, A. Shalev), Progr. Math. 184, Birkhäuser, Boston, MA, 2000, 349-410. Zbl 0973.20043 MR 1765127

[34] C. Weibel, An Introduction to Homological Algebra. Cambridge Stud. Adv. Math. 38, Cambridge University Press, Cambridge 1994. Zbl 0797.18001 MR 1269324

[35] J. S. Wilson, Profinite Groups. London Math. Soc. Monogr. (N.S.) 19, Oxford University Press, New York 1998. Zbl 0909.20001 MR 1691054

Received December 3, 2002; revised June 20, 2005

Peter Symonds, School of Mathematics, University of Manchester, P.O. Box 88, Manchester M60 1QD, England

E-mail: Peter.Symonds@manchester.ac.uk 